\title{
Granite magmatism and crustal evolution of the Shyok-Darbuk corridor of NE Ladakh, India
}

Wadia Institute of Himalayan Geology, 33 General Mahadeo Singh Road, Dehradun - 248001, Uttarakhand, India. E-mail: daga_megha@yahoo.com

Continental growth is episodic, and arc-continental margin has importance for the formation of continental crust in the Shyok Suture Zone (SSZ). To shed light on the petrogenesis of mantle derived orthogneiss and hybrid source derived leucogranites, a detailed geochemical study has been conducted on granitoids which are exposed between the Shyok-Darbuk of the Pangong transpressional zone. The Shyok-Darbuk is a complex zone, marked by migmatization, crustal anatexis, in-situ emplacement of various granitoids within metapelites and orthogneiss. Major and trace element data have been used in this paper to classify the granitoids to estimate the possible source regions and tectonic environment of magma generation and emplacement.

The metaluminous Hbl-Bt orthogneiss (OG) of Eocene to Late-Cretaceous age show chemical characteristics typical of Andean-type granitoids. The late phase granitoids (porphyritic granites, coarse leucogranites, two-mica \pm garnet bearing leucogranite dyke swarms) of Miocene age have generated from the intracrustal partial melting of hybrid magma source, involving predominantly of orthogneiss (derived from mantle source) and subordinate amounts of metapelites (crustal source). The generation of these crustally-derived magma bodies has also been promoted by an influx of externally derived aqueous fluids. Further, the variation of felsic melts from leucogranodiorite (metaluminous) to leucogranite (peraluminous), calls for the geochemical heterogeneity, largely due to protolith internal heterogeneity at source. The leucosome of migmatites and the common leucogranites of the nearby Darbuk pluton are coeval, which record melt flow syntectonically through the deforming crust. The various leucosome magma channels in the migmatites are considered to merge and link-up with each other during their ascent to upper crustal levels, resulting in feeding stocks and plutons. In the Shyok-Darbuk corridor of the Pangong range it is thus envisaged that, the fundamental role in the growth of continental crust, witnessed tectonism that span from initial arc-continent to continent-continent collision regimes.

\section{Introduction}

In the overall convergent framework of India-Asia collision multiple subduction events took place resulting in magmatic episodes of Jurassic-Cretaceous age (Debon et al., 1981, 1987; Le Fort, 1986). Yin and Harrison (2000) suggested that the Kohistan-Ladakh arc collided with the Karakoram terrane during Cretaceous along the Shyok Suture Zone (SSZ), followed by closure of the Neotethyan Ocean along the Indus Tsangpo Suture Zone (ITSZ) and accretion of the composite Karakoram-Kohistan-Ladakh arc terrane with Indian Plate. Intensive isotopic dating indicated that most of the granites in the Karakoram terrane were emplaced during Miocene (Schärer et al., 1990; Phillips et al., 2004; Jain and Singh, 2008), older emplacement is less documented according to the available literature (Searle et al., 1998; Rai and Rameshwar Rao, 2005; Jain and Singh, 2008; Ravikant, 2006), while the voluminous Ladakh Batholith witnessed Late Cretaceous-Paleocene (74-45 Ma) granodioritegranite emplacement in short pulses. According to Ravikant et al. (2009), the time resolved Hf isotopic compositions of magmatic zircons from the granites in the Karakoram and Ladakh batholith show a large variation that indicate their derivation from both ancient and juvenile continental crust. The Karakoram Cretaceous (95-106 Ma) granites were derived from the Karakoram Proterozoic basement, the Cenozoic (56-76 Ma) granites were derived from the juvenile crust, and the Miocene (9-26 Ma) granitic activity involved significant input from the Indian crust.

There are, however, certain isolated metamorphic terrains and litho-units having granites which have different geological significance. Recent workers have attached great importance to this crustal block of the eastern Shyok Suture Zone (SSZ) that is essentially composed of Darbuk-Tangtse granites and metasediments (Rameshwar Rao and Rai, 2003, 2009a; Singh et al., 2004; Ravikant, 2006; Ravikant et al., 2009; Reichardt et al., 2010; Roy et al., 2010). Bounded by two branches of Karakoram Fault on either side, they represent a faulted block detached and transported from the base of the Karakoram batholith during Miocene, referred to as Pangong Range (Searle et al., 1998). The Pangong Range comprises of the calc-alkaline suite of orthogneiss having composition from tonalite to granodiorite, metapelites, calc-silicates and amphibolites. The calc-alkaline granitoids and meta-sedimentary sequence are 
migmatized, giving rise to leucogranite intrusions (Weinberg et al., 2009), however, Sen et al. (2009) have suggested that Pangong Granodiorite to be formed by partial melting of the migmatite.

The Shyok-Darbuk section of the Pangong Range is a key to understand the changing geodynamic processes through time, as magmatism spans from pre-collisional subduction-related calcalkaline orthogneiss to post-collisional related leucogranites, the latter involving anatexis of a hybrid source. Globally, several studies show interaction and mixing of magmas derived from mantle and crustal sources (e.g., Gray, 1984; Hawkesworth and Vollmer, 1979; Andersson, 1991; Castro et al., 1991; Crawford and Searle, 1992; Guillot and Le Fort, 1995; Reichardt et al. 2010). However, a precise field, petrographic and geochemical characterization of the different granitoids and their geodynamic context of emplacement still poorly known. Consequently it is important to characterize magmatic evolution history of different granitoids, and geodynamic context of magma generation and emplacement within the regional geological framework of Himalayan orogeny. Further the relationship of granitoids of Shyok-Darbuk section with Ladakh and Karakoram arc related granitoids is remains elusive. The purpose of the present study is to present field, petrographic and elemental (major and trace) data for the granitoids of Shyok-Darbuk section and to discuss petrogenesis including magmatic evolution and nature of their sources and geodynamic settings of magma generation. Finally, the granitoids magmatism of studied corridor is compared with Ladakh and Karakoram regions to understand the temporal evolution of magmatism through time in changing geodynamic scenario of north western Himalaya.

\section{Geological setting}

The crustal growth in the central Asia is represented by the progressive accretion of continental terranes, with age of accretion decreasing towards south. The Karakoram Plate is an active convergent zone with intense crustal shortening and episodic magmatism occurring for over a period of at least $80 \mathrm{Ma}$. Calcalkaline magmatism to the north of Indus Tsangpo Suture Zone (ITSZ) has resulted into the development of Andean-type Ladakh Batholith (cf. Honeggar et al., 1982; Scharer et al., 1984; Weinberg and Dunlap, 2000; Upadhyay et al., 2008). This large Early Cretaceous to Eocene magmatic arc is bounded to the north by the Shyok Suture Zone (SSZ). The final suturing of the Neotethyan ocean at $\sim 50 \mathrm{Ma}$ (e.g., Petterson and Windley, 1985; Trealoar et al., 1996; Rolland et al., 2002; Clift et al., 2002) was followed by thrusting and crustal thickening which propagated both south of the ITSZ in the Zanskar and High Himalayan Ranges (Searle et al., 1988), and also deposition of Indus molasse along the Indus River (Wu et al., 2007).

The Karakoram Fault (KF) that runs through the Nubra-Shyok valley splays into two branches in the eastern Ladakh, the northeastern 'Pangong fault strand' and southwestern 'Tangtse fault strand' (cf. Searle et al., 1998), with an offset of 40-150 km (cf. Searle and Phillips, 2007). The Pangong strand cuts across to the north end of the Tangtse gorge, the southern part of Pangong lake, while the Tangtse strand runs through Darbuk and Tangtse villages towards Chushul. The region between these fault strands from Agham to Chushul is occupied by rocks of Pangong Range (Fig.1), and is being referred as Pangong Transpressional Zone (PTZ) by Phillips (2008). The Pangong Range exposed between Shyok and Chushul in the eastern SSZ is dotted

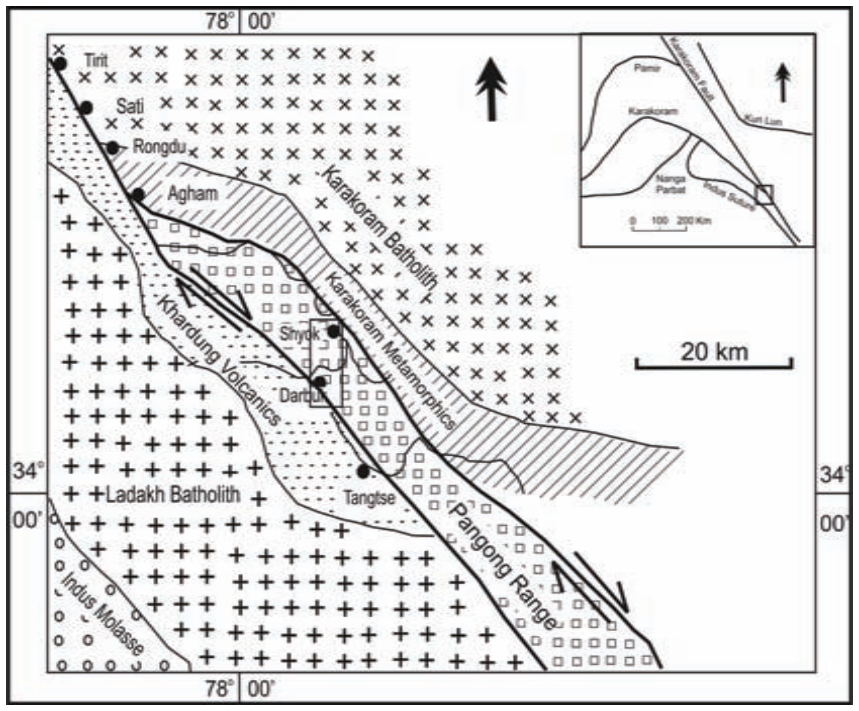

Figure 1. Geological map of eastern Shyok Suture Zone of NW India (simplified from map given in Weinberg and Mark, 2008), showing the location map of Fig.2.

with small granitoid intrusions forming small, isolated plutons and plugs (e.g., Darbuk granite, Tangtse granite). These NW-SE trending granitoid bodies are situated in a peculiar situation between two tectonostratigraphic units belonging to two contrasting terranes, the Pangong Metamorphics (Choudhary, 1983; Virdi, 1981) or Karakoram Metamorphic Complex (Sinha et al., 1999; Weinberg and Dunlap, 2000) of the Eurasian Plate to the north and Shyok ophiolitic mélange (oceanic regime) of SSZ to the south. The granitoids have intrusive relationships with the Ophiolitic mélange and the metamorphics (Rai, 1986, 1997, 2008; Rameshwar Rao and Rai, 2009a).

The Shyok-Darbuk part of the PTZ (Figs. 2 and 3a) is subdivided into (i) Shyok Orthogneiss (OG), (ii) Shyok Mixed Zone, and (iii) Darbuk granite:

The Shyok Orthogneiss (OG) is porphyritic, medium to coarse grained calc-alkaline, titanite bearing Hbl-Bt granitoid (mineral abbreviations after Kretz, 1983). Field studies show that the orthogneiss have basic enclaves within it, similar to those within the Ladakh Batholith. The size and shape of enclave varies widely, ranging from $3-50 \mathrm{~cm}$ in width and more than a meter in length. The enclaves show sharp to diffusive contact with host orthogneiss. From several lines of field and microstructural studies, Kumar and Singh (2008) and Kumar (2010) have suggested that Ladakh Igneous Province (LIP) represents composite mafic-silicic intrusions, formed by the interactions of several batches of coeval mafic and felsic magmas.

In the Shyok-Tangtse region migmatites are associated with leucogranite intrusions (Weinberg and Searle, 1998; Weinberg and Mark, 2008; Weinberg et al., 2009). The Shyok Mixed Zone which starts near Shyok village runs for about 6-8 $\mathrm{km}$ towards Darbuk village. The zone comprises of a variety of migmatites and high-grade metamorphics including amphibolites, orthogneiss, calc-silicates and metapelites which are considered to be lower crustal equivalents of the Pangong metamorphic complex exhuming over $6500 \mathrm{~m}$ during Late Tertiary transpression (18.0 - 11.3 Ma) between the two strands of the Karakoram fault (cf. Searle et al., 1998; Weinberg and Searle, 1998; Phillip, 2008). They are intruded by crustal derived leucosome melts, the leucosomes are linked to leucogranites that form a complex melt flow intrusive sheet network (Fig. 3b and c). The calc-silicates 


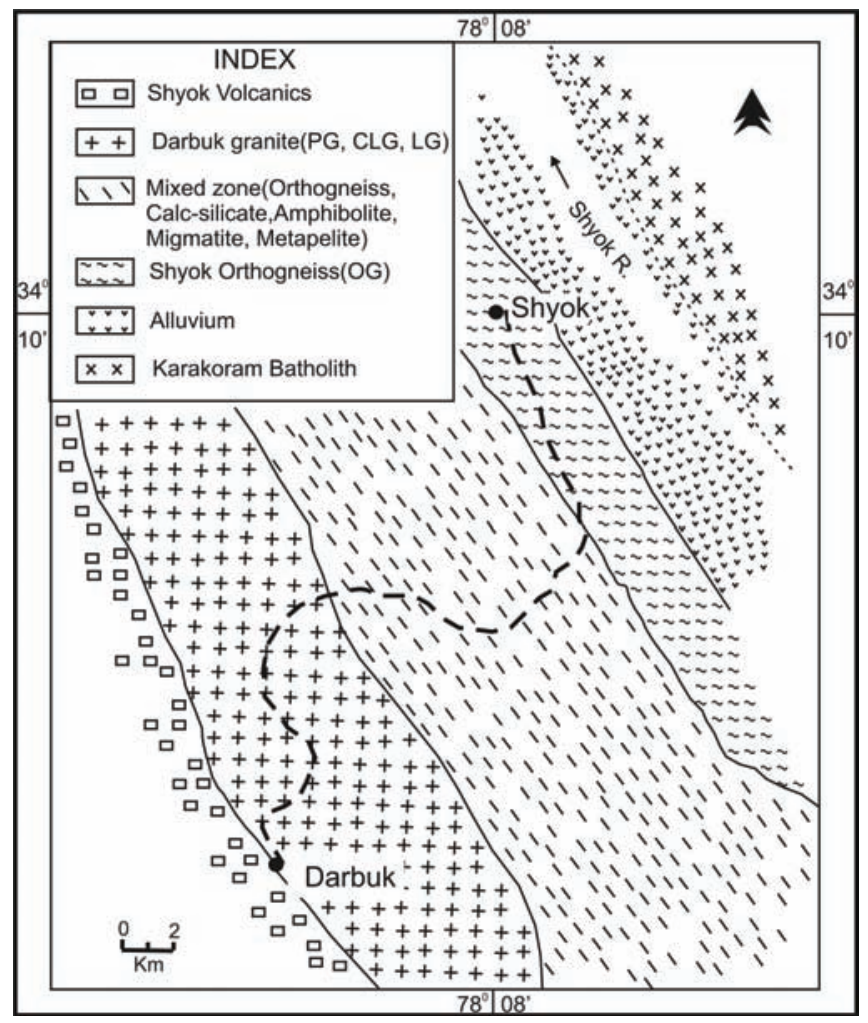

Figure 2. Traverse map showing the dominant rock types along the Shyok-Darbuk corridor. The intrusive contact of Darbuk granitoids and Shyok volcanics and ophiolites is after Rai (1986, 1997, 2008).

of the zone have inter-bedded leucogranite (LG) bands within it (Fig. 3d), and generally contain $\mathrm{Grt}+\mathrm{Sc}+\mathrm{Hbl}+\mathrm{Cal}+\mathrm{Pl}+\mathrm{Qtz}+\mathrm{Spn}$. The metapelites, on the other hand, have Grt+Pl+Qtz+Sil/Ky+ $\mathrm{B} t \pm \mathrm{Ms} \pm \mathrm{Kfs}+\mathrm{Ap}+\mathrm{Ep}$ assemblage. The other rock type the mixed zone, the OG, is compositionally similar to that of Shyok orthogneiss, and show basic enclaves of amphibolites and amphibolite schists (Fig. 3e). The leucogranites of the mixed zone although cross-cutting, dyke-like sheets developed locally (Fig. 3c), most sheets intruded parallel to the foliation (Fig. 3d). The coarse pegmatite also occurs, and show isolated blocks of OG within it (Figs. 3f). The LG patches with diffuse boundaries with the host OG form an irregular network of $\mathrm{Bt} \pm \mathrm{Grt} L \mathrm{~L}$ pockets and veins, feeding into larger $\mathrm{LG}$ with width varying up to one meter. Leucogranite dykes and sills are partially mylonitized and deformed (Fig. 3g).

In the Shyok-Darbuk section outside the mixed zone one can see ubiquitous presence of Ms-Bt \pm Grt LG in form of dykes, stocks and plutons of tens to hundreds of meters size, referred to as Darbuk Granite (Figs. 2 and 3a). It extends up to Tangtse, and is intrusive into the Shyok Ophiolitic mélange, all along its southern margin (cf. Rai, 1986, 2008). The LG show variation from Porphyritic Granites (PG), Coarse Leucogranites (CLG) to Leucogranites \pm garnet (LG). The late phase granitoids, PG and CLG, show intrusive contact with OG and metapelites along the northern margin. They also show intrusive relations with one another and are intruded by a younger phase of LG (Figs. 3h-j). Weinberg and Searle (1998) consider the Tangtse LG to be part of the Karakoram batholith, and that the LG were emplaced just before or during the early stages of movement on the Karakoram fault from the melts extracted from the crustal rocks during continental collision.

\section{Petrography}

The OG is distinguishable in having higher concentration of plagioclase, hornblende, biotite and sphene, where hornblende forms straight rhombic to prismatic crystals sometime showing twinning (Fig. 4a). Euhedral to subhedral oligoclase constitute majority of the phenocrysts, show twinning and normal to oscillatory zoning (Fig. 4b). Sphene generally occurs in rhombic form with prominent parting, lamellar twining (Fig. 4c), and with quartz and feldspar inclusions, while zircons and apatite occur in euhedral to subhedral forms. The PG, CLG and LG have phenocrysts, mainly of plagioclase, along with microperthite and quartz. Euhedral to subhedral plagioclase is twinned and show a variety of zoning, ranging from normal, oscillatory (Fig. 4d) to patch type. Feldspars are often characterized by myrmekite and perthite intergrowth, and show brittle to ductile microstructures. Some of the feldspar megacrysts show parallel pull-apart structure, the openings of which are filled with recrystallized quartz and feldspar (Fig. 4e). Mylonitized rocks exhibit a characteristic core-mantle mortar structure in which isolated quartz and feldspar porphyroclast occur within fine-grained matrix of recrystallized feldspar, quartz and mica flakes. K-feldspar occurs as subhedral grains, occasionally showing Carlsbad twinning (Fig. 4f). Microperthites often show poikilitic structure and has inclusions of euhedral to subhedral twinned plagioclase, biotite and quartz (Fig. 4g). These inclusions have crystallized at earlier stage at high temperature as compared to enclosing feldspar, therefore they are stable at low temperature when feldspar crystallized, hence are not reacted, but may change their shapes to minimize the interfacial free energy of their boundaries with the phenocryst. Quartz is subhedral to anhedral and shows evidence of crystal-plastic deformation in the form of ribbon structures, chess board structures and undulose extinction. Micas, both muscovite and biotite, always constitute less than $5 \%$. They show fish structure of the type Group 2 (after ten Grotenhuis et al., 2003) which occur discretely (Fig. 4h). As compare with CLG and PG, the LG have exceptionally low abundance of muscovite and biotite. Scanty garnet is observed in coarse as well as medium grained varieties. Accessory minerals include euhedral apatite grains along with zircon as inclusions in feldspar and quartz, along with minor amounts of sphene and epidote.

\section{Geochemistry}

A representative selection of Hbl-Bt Orthogneiss (OG), Porphyritic Granites (PG), Coarse Leucogranites (CLG), and Leucogranites with or without garnet (LG) collected from widely separated localities along the Shyok-Darbuk section of the SSZ, NE Ladakh were analyzed for their major and trace elements at Wadia Institute of Himalayan Geology, Dehradun. The major and trace elemental analyses of 76 samples were carried out on pressed pellets using XRF (SIEMENS SRS 3000) instrument, calibrated against both national and international standards. Analytical accuracy on XRF is better than $5 \%$ for major oxides and $12 \%$ for trace elements, and the precision in terms of maximum observed standard deviation on repeated measurements is better than $2 \%$ (Saini et al., 1998). A subset of 30 samples were also analyzed for REE using ICP-MS (PerkinsElmer SCIEX). The relative standard deviation (RSD) for most REE elements analyzed on ICP-MS is better than 10\% (Khanna et al., 2009). Major and trace element data are presented in Tables 1 to 3, 

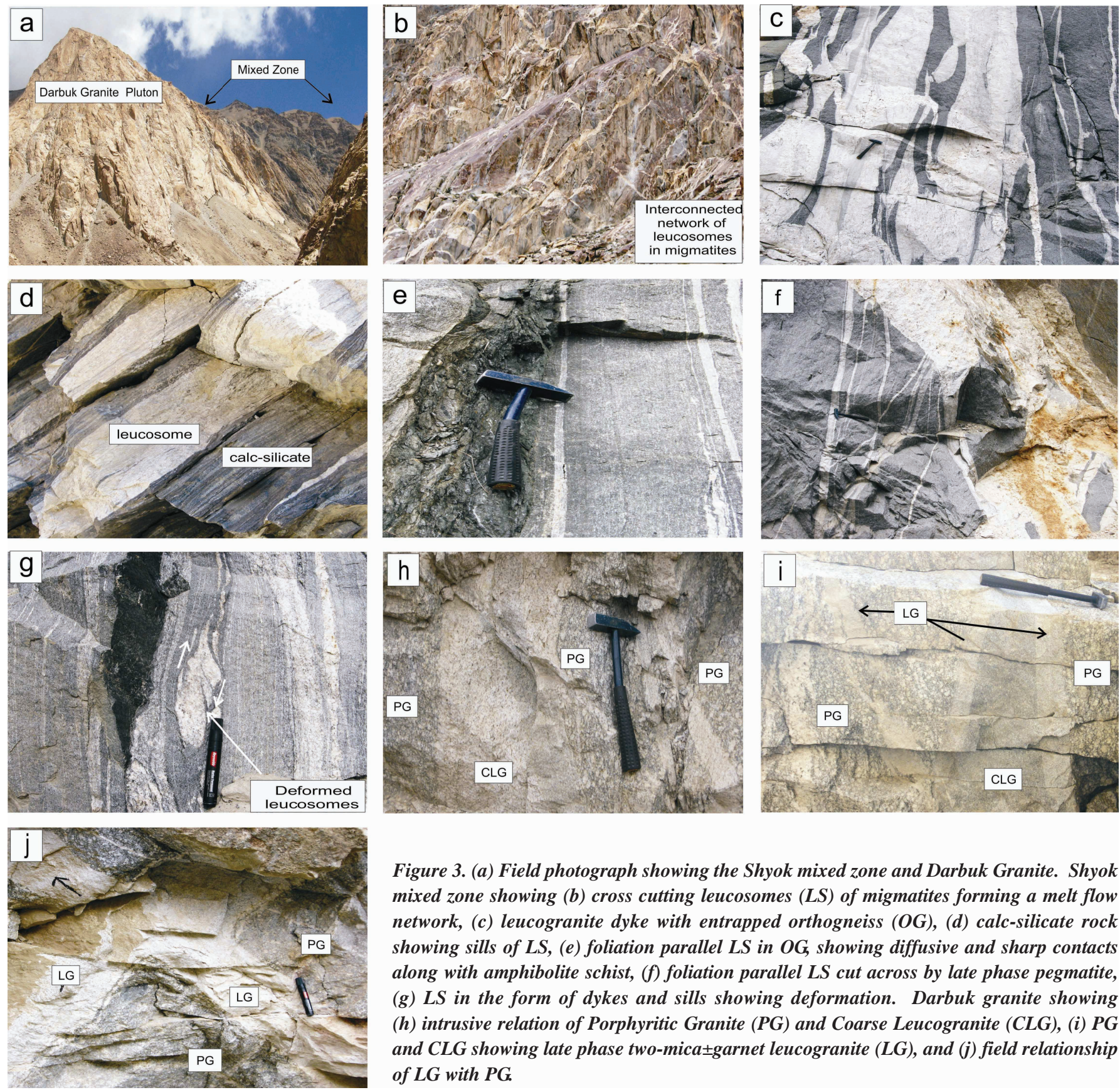

Figure 3. (a) Field photograph showing the Shyok mixed zone and Darbuk Granite. Shyok mixed zone showing (b) cross cutting leucosomes (LS) of migmatites forming a melt flow network, (c) leucogranite dyke with entrapped orthogneiss $(O G),(d)$ calc-silicate rock showing sills of $L S$, (e) foliation parallel $L S$ in $O G$, showing diffusive and sharp contacts along with amphibolite schist, (f) foliation parallel LS cut across by late phase pegmatite, (g) LS in the form of dykes and sills showing deformation. Darbuk granite showing (h) intrusive relation of Porphyritic Granite (PG) and Coarse Leucogranite (CLG), (i) PG and CLG showing late phase two-mica \pm garnet leucogranite $(L G)$, and (j) field relationship of $L G$ with $P G$.

and the mean geochemical values along with their ranges (major, trace and REE) of each individual rock-type is given in Table 4. To characterize the compositional types, petrogenetic processes and finger printing tectonic settings, the plotting of geochemical data is done on different variation diagrams encompassing both major and trace elements (Figs. 5-11). The geochemistry of the early OG and the late phase (PG, CLG and LG) granitoids show fractionation relations on variation diagrams, suggesting for some genetic linkage, hence are discussed together.

The OG is mesocratic and is less evolved among the rock-types studied (Table 4). They are characterized by tonalite to granodiorite composition, calc-alkaline nature, and show metaluminous nature (Figs.5a-b; Table 4). On the one hand, the PG, CLG and LG are leucocratic and are moderately to highly evolved. They are characterized by granodiorite to granite compositions, calc-alkaline nature (Figs. 3a-b), and metaluminous to peraluminous affinity (Figs. 5a-b; Table 4). The OG together with PG, CLG and LG show generally coherent (enriched or depleted) variation on Harker's binary diagrams. They show negative correlation in case of $\mathrm{CaO}, \mathrm{FeO}^{\mathrm{t}}, \mathrm{MgO}, \mathrm{MnO}, \mathrm{Al}_{2} \mathrm{O}_{3}$, $\mathrm{P}_{2} \mathrm{O}_{5}, \mathrm{TiO}_{2}, \mathrm{Sr}, \mathrm{Cr}, \mathrm{Ni}, \mathrm{La}, \mathrm{Ce}$ and $\mathrm{Zr}$ against $\mathrm{SiO}_{2}$. However, elements like $\mathrm{Na}_{2} \mathrm{O}, \mathrm{K}_{2} \mathrm{O}, \mathrm{Rb}$ and mol. A/CNK shows a positive correlation against $\mathrm{SiO}_{2}$. Some of these trends are depicted in Fig.6a. The chondrite normalized pattern of these granitoids represented in Fig.7a exhibit enriched LREE pattern relative to HREE, along with variable negative Eu anomalies. The PG and CLG show fractionated REE pattern with conspicuous negative Eu anomaly, while the more evolved garnet-bearing LG have low abundance of total REE, show enriched HREE pattern with less fractionated $(\mathrm{La} / \mathrm{Lu})_{\mathrm{N}}$ ratios, and appreciable high Eu/Eu* ratios (Table 4). In contrast, the early phase OG have highly fractionated trends with no significant Eu anomaly $\left(\mathrm{Eu} / \mathrm{Eu}^{*}\right.$ 

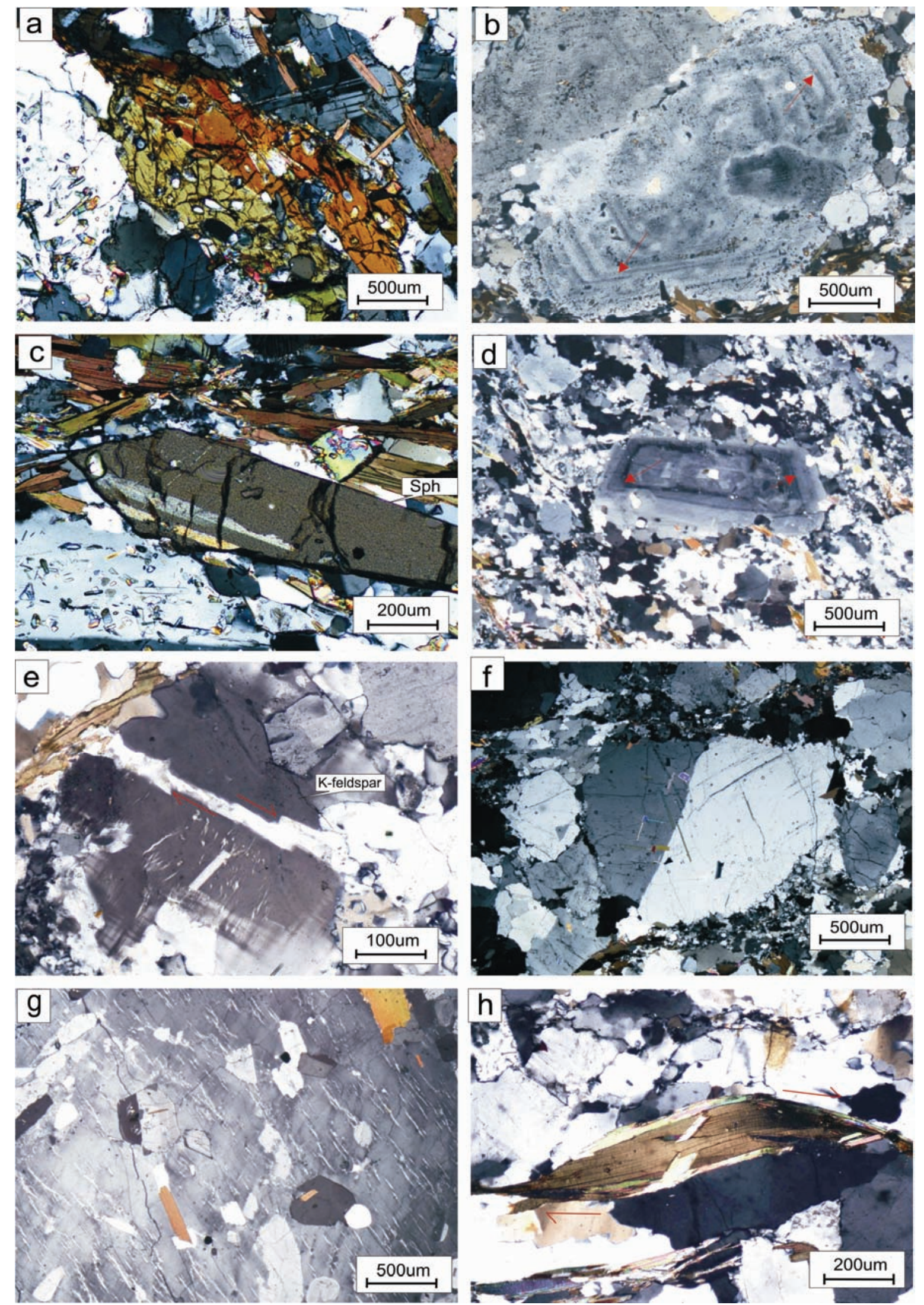

Figure 4. (a) OG showing staught rhombic to prismatic twinned crystal of $\mathrm{Hbl}$, (b) plagioclase phenocryst of OG showing oscillatory zoning, (c) rhombic parting and lamellar twinning in sphene from $O G$, (d) PG showing euhedral oligoclase plagioclase having sodic margins, (e) pull-apart structure of feldspar in CLG, with recrystallized quartz and feldspar, $(f)$ PG showing Carlsbard twinning in orthoclase, (g) microperthite in PG showing poikilitic structure with inclusions of euhedral to subhedral twinned plagioclase, biotite and quartz, and (h) CLG showing Type Group 2 Mica fish structure. 


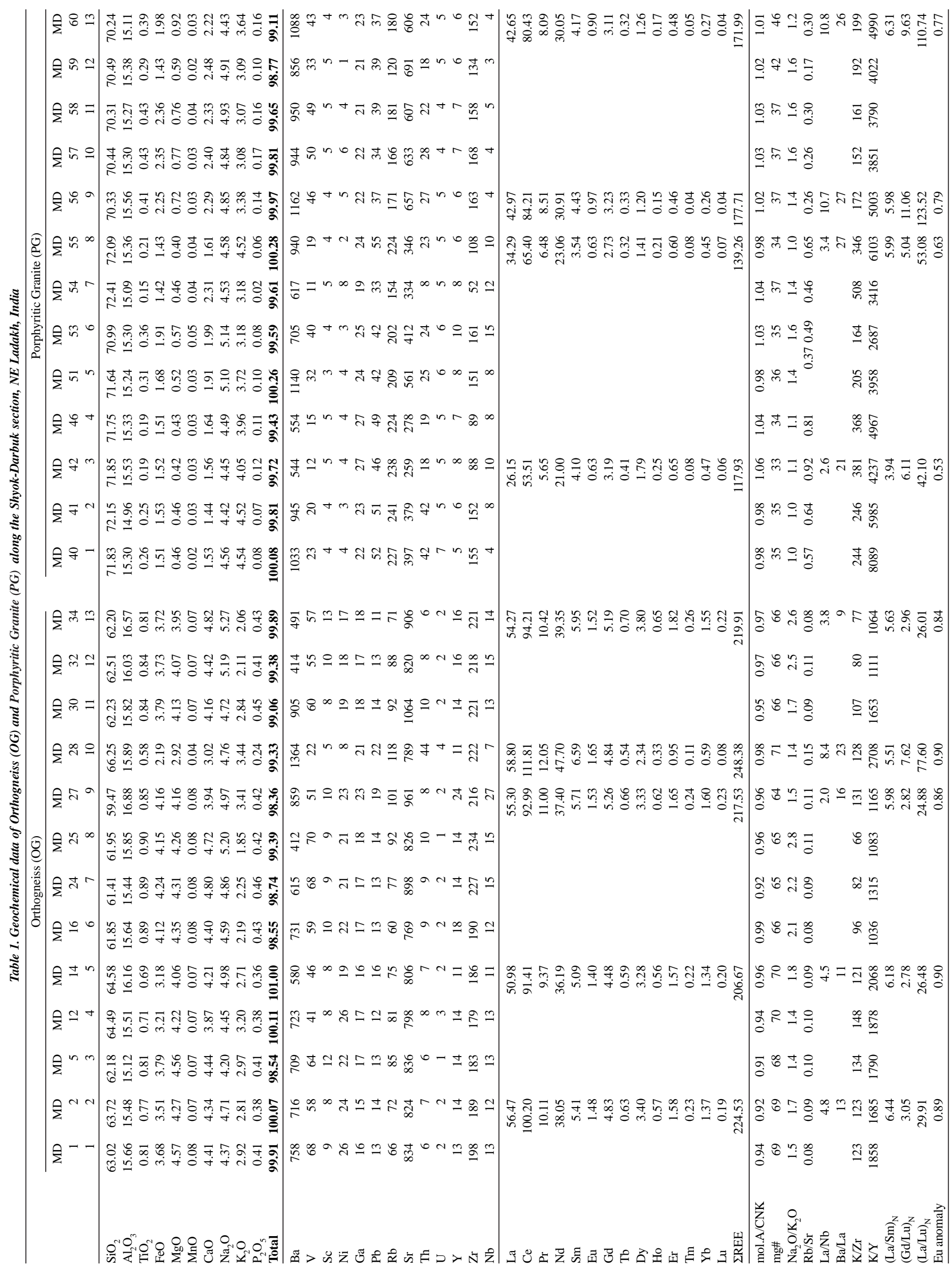


Table 2. Geochemical data of Coarse Leucogranite (CLG) along Shyok-Darbuk Section, NE Ladakh, India

\begin{tabular}{|c|c|c|c|c|c|c|c|c|c|c|c|c|c|c|c|c|c|c|c|c|c|}
\hline & MD & MD & MD & MD & MD & MD & MD & MD & MD & MD & MD & MD & MD & MD & MD & MD & MD & MD & MD & MD & MD \\
\hline & 21 & 33 & 45 & 47 & 48 & 52 & 67 & 69 & $15 \mathrm{~A}$ & 139 & 140 & 141 & 142 & 143 & 144 & 145 & 146 & 147 & 148 & 149 & 150 \\
\hline & 1 & 2 & 3 & 4 & 5 & 6 & 7 & 8 & 9 & 10 & 11 & 12 & 13 & 14 & 15 & 16 & 17 & 18 & 19 & 20 & 21 \\
\hline $\mathrm{SiO}_{2}$ & 73.88 & 73.96 & 73.24 & 74.01 & 73.67 & 74.15 & 73.35 & 72.95 & 73.90 & 74.09 & 72.56 & 73.48 & 73.05 & 73.10 & 73.99 & 74.70 & 74.53 & 74.64 & 74.69 & 75.45 & 73.36 \\
\hline $\mathrm{Al}_{2} \mathrm{O}_{3}$ & 14.26 & 14.67 & 14.58 & 13.89 & 14.53 & 14.51 & 15.08 & 14.82 & 14.67 & 14.34 & 15.29 & 14.15 & 15.01 & 14.84 & 14.45 & 14.54 & 14.95 & 14.80 & 14.79 & 14.63 & 15.08 \\
\hline $\mathrm{TiO}_{2}$ & 0.16 & 0.06 & 0.09 & 0.10 & 0.09 & 0.05 & 0.03 & 0.11 & 0.06 & 0.06 & 0.08 & 0.08 & 0.08 & 0.07 & 0.08 & 0.05 & 0.08 & 0.05 & 0.05 & 0.04 & 0.05 \\
\hline $\mathrm{FeO}^{2}$ & 1.03 & 0.56 & 1.30 & 1.28 & 0.97 & 0.70 & 0.35 & 0.87 & 0.69 & 0.52 & 0.81 & 0.83 & 0.76 & 0.75 & 0.77 & 0.66 & 0.79 & 0.66 & 0.67 & 0.57 & 0.70 \\
\hline $\mathrm{MgO}$ & 0.41 & 0.23 & 0.27 & 0.37 & 0.27 & 0.18 & 0.20 & 0.40 & 0.25 & 0.29 & 0.32 & 0.32 & 0.31 & 0.27 & 0.30 & 0.23 & 0.06 & 0.23 & 0.23 & 0.21 & 0.24 \\
\hline $\mathrm{MnO}$ & 0.01 & 0.01 & 0.03 & 0.04 & 0.03 & 0.02 & 0.02 & 0.02 & 0.02 & 0.03 & 0.02 & 0.03 & 0.03 & 0.03 & 0.03 & 0.03 & 0.03 & 0.03 & 0.03 & 0.03 & 0.04 \\
\hline $\mathrm{CaO}$ & 1.74 & 1.38 & 1.87 & 1.95 & 1.76 & 1.60 & 1.85 & 2.18 & 1.31 & 1.80 & 1.53 & 1.60 & 1.65 & 1.59 & 1.60 & 1.47 & 1.59 & 1.42 & 1.45 & 1.42 & 1.43 \\
\hline $\mathrm{Na}_{2} \mathrm{O}$ & 3.66 & 3.73 & 4.42 & 4.21 & 4.36 & 4.56 & 4.34 & 4.00 & 4.13 & 4.08 & 3.71 & 3.83 & 4.00 & 4.06 & 3.91 & 4.17 & 4.06 & 4.16 & 4.16 & 4.26 & 4.23 \\
\hline $\mathrm{K}_{2} \mathrm{O}$ & 4.75 & 5.46 & 3.58 & 3.73 & 3.88 & 4.14 & 4.67 & 4.11 & 5.20 & 3.82 & 4.88 & 3.98 & 4.08 & 4.00 & 4.24 & 4.27 & 4.20 & 4.29 & 4.33 & 4.19 & 4.23 \\
\hline $\mathrm{P}_{2} \mathrm{O}_{5}$ & 0.10 & 0.02 & 0.03 & 0.03 & 0.05 & 0.01 & 0.03 & 0.03 & 0.05 & 0.04 & 0.13 & 0.05 & 0.13 & 0.09 & 0.05 & 0.04 & 0.05 & 0.04 & 0.10 & 0.10 & 0.07 \\
\hline Total & 100.0 & 100.1 & 99.41 & 99.61 & 99.62 & 99.93 & 99.92 & 99.49 & 100.3 & 99.07 & 99.32 & 98.36 & 99.10 & 98.81 & 99.41 & 100.2 & 100.3 & 100.3 & 100.5 & 100.9 & 99.43 \\
\hline $\mathrm{Ba}$ & 2188 & 1502 & 619 & 703 & 715 & 566 & 432 & 881 & 379 & 689 & 595 & 527 & 617 & 556 & 607 & 448 & 599 & 432 & 419 & 394 & 421 \\
\hline V & 16 & 0 & 1 & 6 & 4 & 3 & 3 & 7 & 9 & 1 & 1 & 4 & 29 & 28 & 3 & 3 & 1 & 1 & 49 & 65 & 6 \\
\hline $\mathrm{Sc}$ & 4 & 4 & 5 & 4 & 5 & 3 & 4 & 5 & 4 & 4 & 7 & 8 & 4 & 5 & 6 & 4 & 5 & 4 & 4 & 4 & 3 \\
\hline $\mathrm{Ni}$ & 1 & 0 & 4 & 3 & 3 & 4 & 3 & 0 & 2 & 0 & 1 & 1 & 2 & 1 & 0 & 0 & 0 & 1 & 1 & 0 & 1 \\
\hline $\mathrm{Ga}$ & 13 & 15 & 20 & 18 & 22 & 24 & 20 & 18 & 17 & 20 & 25 & 22 & 23 & 23 & 21 & 23 & 22 & 23 & 23 & 24 & 24 \\
\hline $\mathrm{Pb}$ & 24 & 38 & 48 & 42 & 49 & 42 & 48 & 44 & 27 & 47 & 55 & 49 & 50 & 49 & 51 & 56 & 52 & 55 & 54 & 58 & 58 \\
\hline $\mathrm{Rb}$ & 91 & 110 & 190 & 188 & 188 & 209 & 128 & 100 & 127 & 164 & 236 & 188 & 182 & 184 & 178 & 205 & 179 & 211 & 209 & 199 & 208 \\
\hline $\mathrm{Sr}$ & 625 & 566 & 249 & 268 & 265 & 561 & 260 & 349 & 187 & 273 & 207 & 207 & 226 & 207 & 218 & 177 & 221 & 175 & 173 & 171 & 172 \\
\hline Th & 10 & 9 & 8 & 5 & 14 & 25 & 4 & 11 & 8 & 7 & 10 & 10 & 8 & 11 & 9 & 7 & 10 & 9 & 8 & 6 & 10 \\
\hline $\mathrm{U}$ & 4 & 5 & 5 & 55 & 6 & 6 & 5 & 6 & 6 & 6 & 6 & 5 & 5 & 5 & 6 & 5 & 6 & 7 & 6 & 7 & \\
\hline $\mathrm{Y}$ & 6 & 5 & 11 & 7 & 11 & 8 & 8 & 9 & 6 & 9 & 8 & 11 & 10 & 10 & 10 & 9 & 10 & 11 & 10 & 11 & 13 \\
\hline $\mathrm{Zr}$ & 63 & 67 & 62 & 61 & 53 & 151 & 37 & 55 & 54 & 38 & 52 & 44 & 44 & 43 & 48 & 38 & 48 & 38 & 40 & 34 & 40 \\
\hline $\mathrm{Nb}$ & 2 & 2 & 12 & 9 & 10 & 8 & 6 & 5 & 4 & 7 & 19 & 10 & 8 & 9 & 8 & 9 & 8 & 9 & 9 & 8 & 9 \\
\hline $\mathrm{La}$ & & 12.65 & & & & & & & & & 19.17 & & 21.78 & & & & & & & 13.64 & \\
\hline $\mathrm{Ce}$ & & 26.08 & & & & & & & & & 40.16 & & 45.49 & & & & & & & 28.13 & \\
\hline $\operatorname{Pr}$ & & 2.81 & & & & & & & & & 4.44 & & 5.04 & & & & & & & 3.07 & \\
\hline $\mathrm{Nd}$ & & 11.10 & & & & & & & & & 17.03 & & 19.95 & & & & & & & 11.98 & \\
\hline $\mathrm{Sm}$ & & 2.20 & & & & & & & & & 3.82 & & 3.73 & & & & & & & 2.91 & \\
\hline $\mathrm{Eu}$ & & 0.65 & & & & & & & & & 0.83 & & 0.93 & & & & & & & 0.64 & \\
\hline $\mathrm{Gd}$ & & 1.57 & & & & & & & & & 3.25 & & 3.04 & & & & & & & 2.56 & \\
\hline $\mathrm{Tb}$ & & 0.17 & & & & & & & & & 0.43 & & 0.37 & & & & & & & 0.35 & \\
\hline Dy & & 0.69 & & & & & & & & & 2.01 & & 1.65 & & & & & & & 1.80 & \\
\hline Ho & & 0.09 & & & & & & & & & 0.29 & & 0.23 & & & & & & & 0.28 & \\
\hline $\mathrm{Er}$ & & 0.23 & & & & & & & & & 0.67 & & 0.58 & & & & & & & 0.75 & \\
\hline $\mathrm{Tm}$ & & 0.03 & & & & & & & & & 0.09 & & 0.07 & & & & & & & 0.12 & \\
\hline $\mathrm{Yb}$ & & 0.16 & & & & & & & & & 0.48 & & 0.38 & & & & & & & 0.74 & \\
\hline $\mathrm{Lu}$ & & 0.03 & & & & & & & & & 0.06 & & 0.05 & & & & & & & 0.10 & \\
\hline$\Sigma \mathrm{REE}$ & & 58.44 & & & & & & & & & 92.72 & & 103.3 & & & & & & & 67.06 & \\
\hline mol.A/CNK & 0.95 & 0.94 & 1.02 & 0.97 & 1.00 & 0.96 & 0.95 & 0.99 & 0.93 & 1.01 & 1.02 & 1.03 & 1.05 & 1.05 & 1.01 & 1.00 & 1.04 & 1.02 & 1.02 & 1.01 & 1.04 \\
\hline $\mathrm{mg} \#$ & 41 & 43 & 28 & 34 & 33 & 31 & 51 & 45 & 40 & 50 & 41 & 41 & 42 & 39 & 41 & 39 & 12 & 39 & 38 & 40 & 38 \\
\hline $\mathrm{Na}_{2} \mathrm{O} / \mathrm{K}_{2} \mathrm{O}$ & 0.8 & 0.7 & 1.2 & 1.1 & 1.1 & 1.1 & 0.9 & 1.0 & 0.8 & 1.1 & 0.8 & 1.0 & 1.0 & 1.0 & 0.9 & 1.0 & 1.0 & 1.0 & 1.0 & 1.0 & 1.0 \\
\hline $\mathrm{Rb} / \mathrm{Sr}$ & 0.15 & 0.19 & 0.76 & 0.70 & 0.71 & 0.37 & 0.49 & 0.29 & 0.68 & 0.60 & 1.14 & 0.90 & 0.80 & 0.89 & 0.82 & 1.16 & 0.81 & 1.20 & 1.21 & 1.16 & 1.21 \\
\hline $\mathrm{La} / \mathrm{Nb}$ & & 5.9 & & & & & & & & & 1.0 & & 2.7 & & & & & & & 1.7 & \\
\hline $\mathrm{Ba} / \mathrm{La}$ & & 119 & & & & & & & & & 31 & & 28 & & & & & & & 29 & \\
\hline $\mathrm{K} / \mathrm{Zr}$ & 626 & 680 & 478 & 510 & 603 & 228 & 1059 & 625 & 800 & 835 & 781 & 756 & 766 & 780 & 732 & 924 & 722 & 926 & 901 & 1015 & 878 \\
\hline $\mathrm{K} / \mathrm{Y}$ & 6319 & 8258 & 2588 & 4153 & 2978 & 4407 & 4902 & 3926 & 7125 & 3635 & 5044 & 3074 & 3367 & 3211 & 3374 & 3787 & 3597 & 3198 & 3447 & 3296 & 2745 \\
\hline$(\mathrm{La} / \mathrm{Sm})_{\mathrm{N}}$ & & 3.55 & & & & & & & & & 3.10 & & 3.61 & & & & & & & 2.89 & \\
\hline$(\mathrm{Gd} / \mathrm{Lu})_{\mathrm{N}}$ & & 5.57 & & & & & & & & & 6.50 & & 7.88 & & & & & & & 3.11 & \\
\hline$(\mathrm{La} / \mathrm{Lu})_{\mathrm{N}}$ & & 37.72 & & & & & & & & & 32.15 & & 47.39 & & & & & & & 13.90 & \\
\hline Eu anomaly & & 1.07 & & & & & & & & & 0.73 & & 0.85 & & & & & & & 0.72 & \\
\hline
\end{tabular}

0.9). The compatible and incompatible elements of PG, CLG and LG have similar pattern on the spider diagram when normalized to Primordial Mantle after Wood et al. (1979). They, in comparison to OG, show relative enrichment of incompatible elements and depletion of less incompatible elements, with distinct negative anomalies of $\mathrm{Nb}, \mathrm{Sr}, \mathrm{P}$ and $\mathrm{Ti}$ (Fig. 7b). The chemistry of granitoids when plotted on $\mathrm{SiO}_{2}$ vs. $\mathrm{Rb} / \mathrm{Zr}$ (Fig. 8) tectonic discrimination diagram after Harris et al. (1986), the samples plot within the volcanic arc granite field, with few samples of CLG and LG spilling over to syn-collision granite field. Further, the understanding of the arc signature of the granitoids on the $\mathrm{Nb}$ and $\mathrm{Y}$ vs $\mathrm{Rb} / \mathrm{Zr}$ binary plots (Fig. 9) after Brown et al. (1984), suggests that the OG were formed in an environment of primitive island arc, while the PG granitoids show their formation from a more mature continental arc environment. However, the CLG and $\mathrm{LG}$ because of their high $\mathrm{Rb}$ and low $\mathrm{Zr}$ have high $\mathrm{Rb} / \mathrm{Zr}$ ratio, and show scattering around mature continental arc field. A feature which is also reflected, in their $\mathrm{Na}_{2} \mathrm{O} / \mathrm{K}_{2} \mathrm{O}$ ratio, wherein the ratio increases with increasing arc maturity. The primitive island arcs have $\mathrm{Na}_{2} \mathrm{O} \%>\mathrm{K}_{2} \mathrm{O} \%$ and hence have high $\mathrm{Na}_{2} \mathrm{O} / \mathrm{K}_{2} \mathrm{O}$ ratio, which decreases with maturity of the arc, and in very mature island arcs this ratio is least. The CLG and LG have low mean $\mathrm{Na}_{2} \mathrm{O} / \mathrm{K}_{2} \mathrm{O}$ ratio of $\sim 1.0$, while the OG have $\sim 1.9$, and the PG has intermediate value of $\sim 1.3$ (Table 4). The geochemical data used for tectonic discrimination using the multi-element parameters (Fig. 10), suggests pre-plate collision for the early phase OG granitoids, and late-orogenic to syncollision for the late phase (PG, CLG and LG) granitoids. 


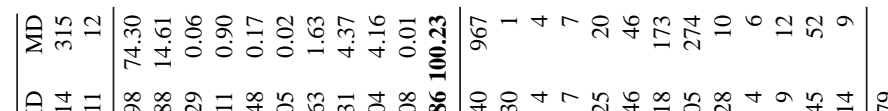

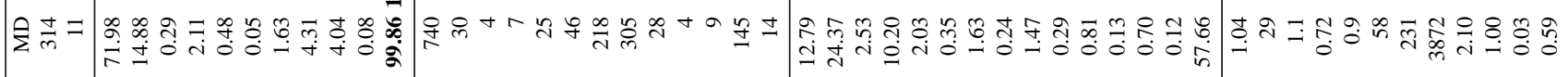

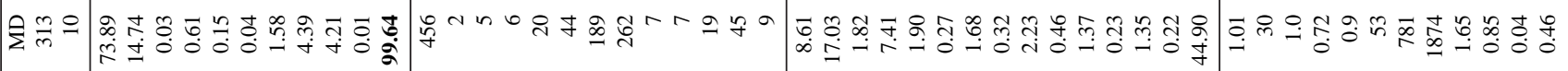

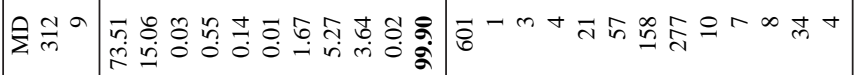

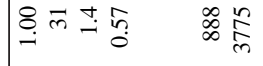

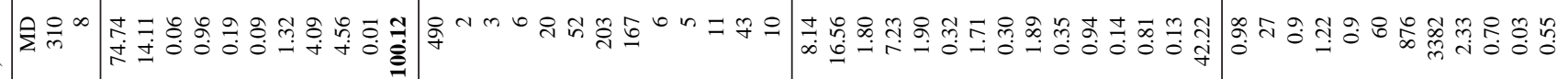

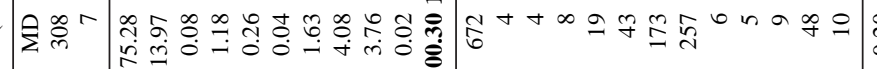

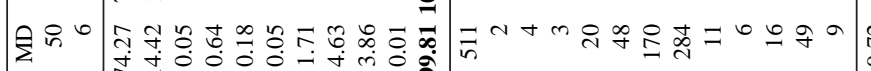

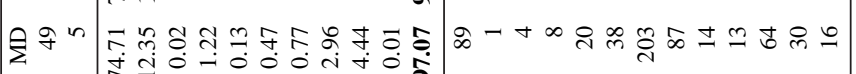

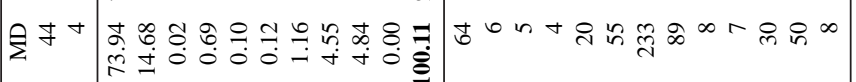

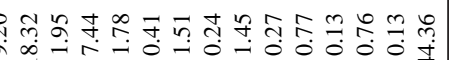

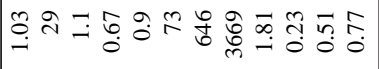

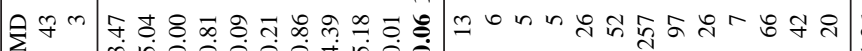

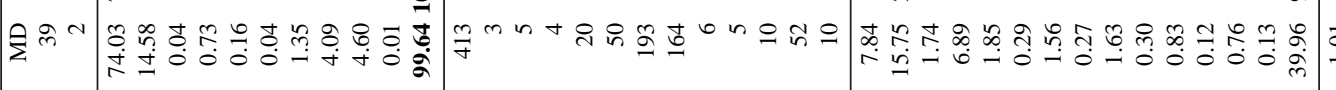

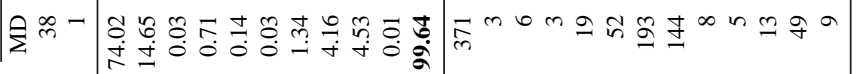

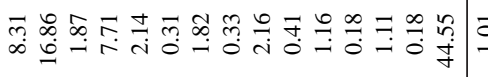

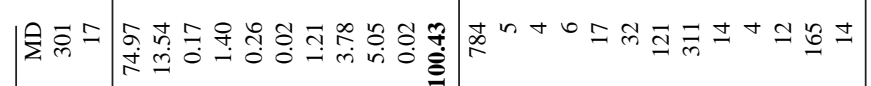

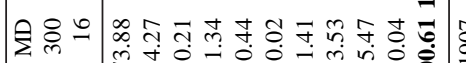

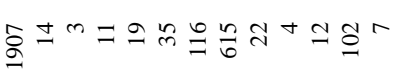

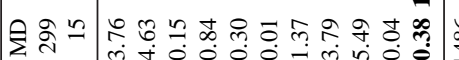

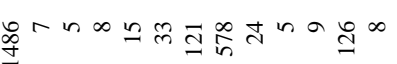

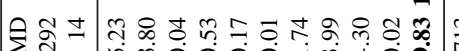

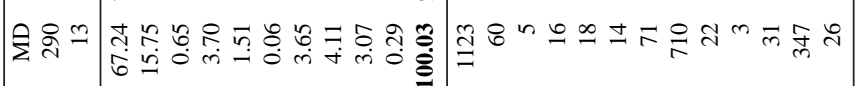

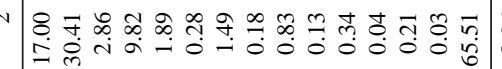

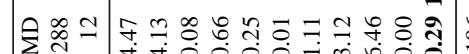

ค

-

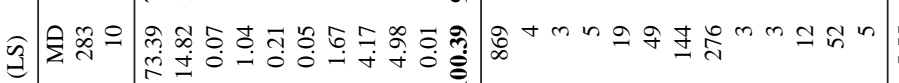

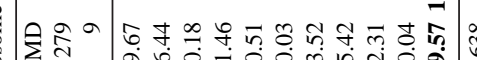

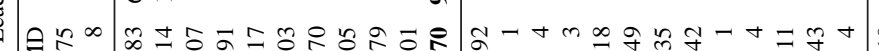

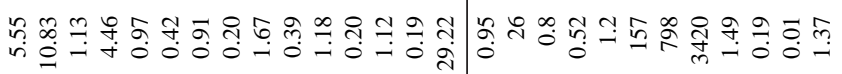

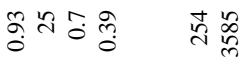

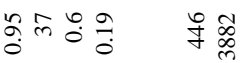

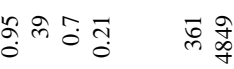

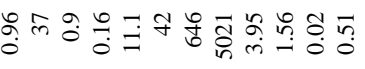

육ำ융

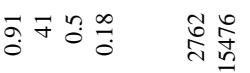

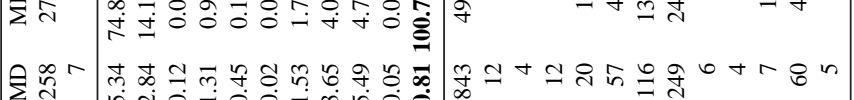

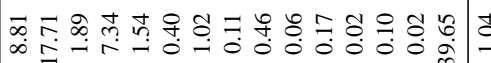

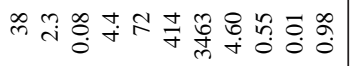

을 \&

$\sum$ y

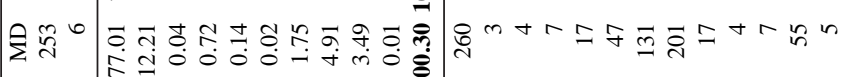

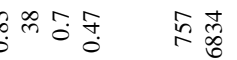

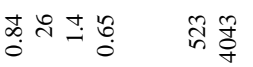

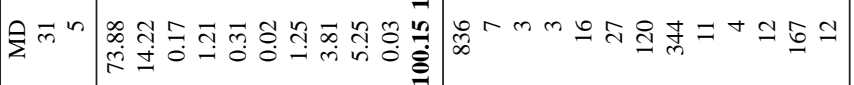

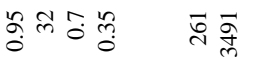

令全

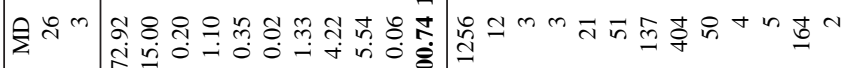

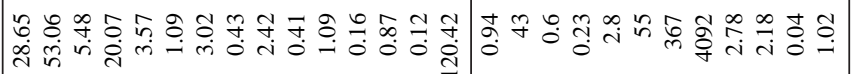

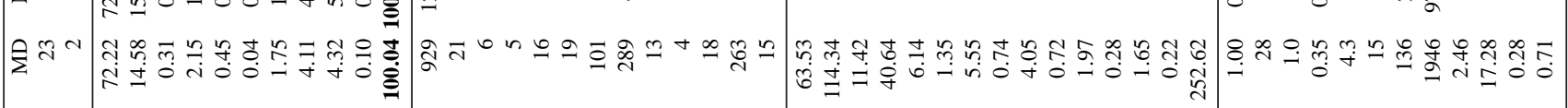

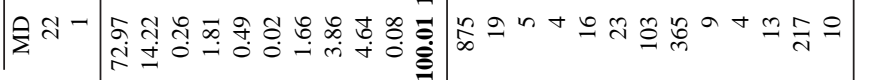

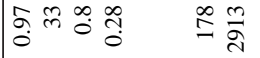

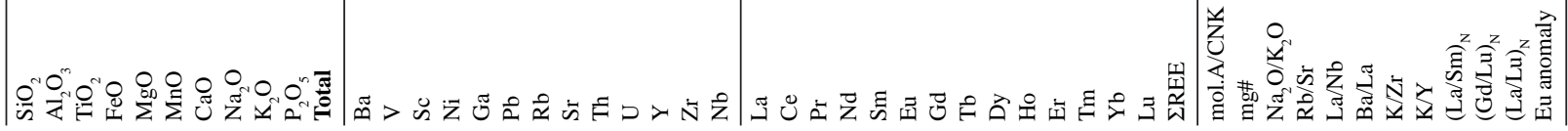




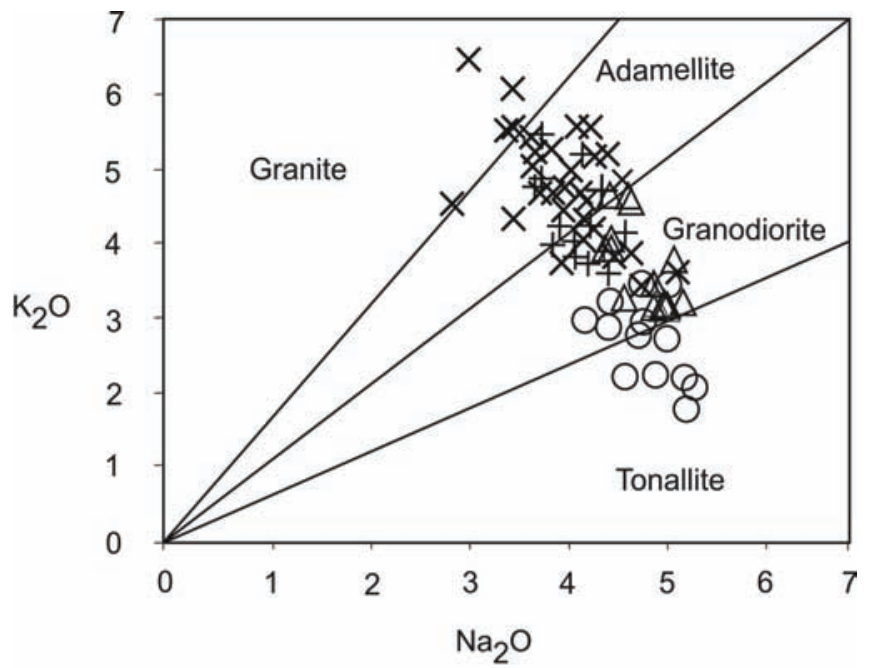

(a)

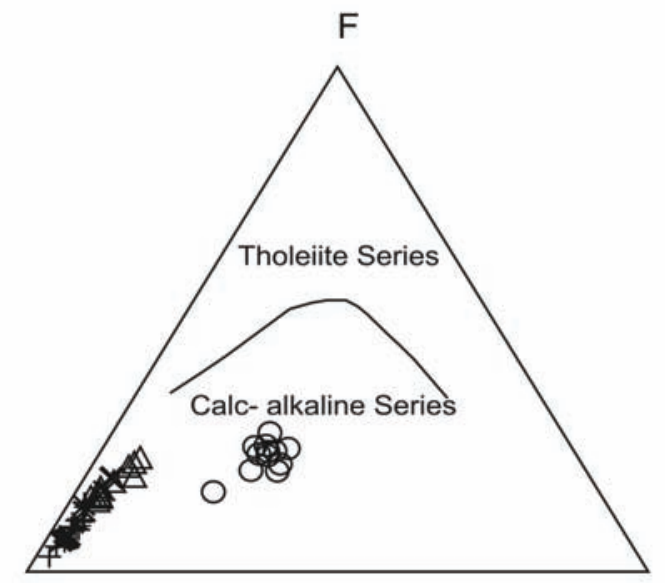

A M

(b)

Figure 5. (a) Classification of rocks on the $\mathrm{Na}_{2} \mathrm{O}$ vs $\mathrm{K}_{2} \mathrm{O}$ binary diagram, fields after Harpum (1963), and (b) AFM diagram showing the plotting of samples in calc-alkaline field (after Barker and Arth, 1976). Symbols: (i) Orthogneiss (OG) - 'open circle'; (ii) Porphyritic Granite (PG) - 'open triangle'; (iii) Coarse Leucogranite (CLG) - 'plus'; and (iv) Leucogranite (LG) - 'cross'.

\section{Leucosome (LS) of mixed zone vs Leucogranites (LG) of Darbuk Granite}

The OG and meta-sedimentary sequence are migmatized, giving rise to leucosome (LS) melts (Weinberg et al. 2009) in the mixed zone (Fig. 3a). They show compositional variation from granodiorite to granite (Fig. 5a; Table 3), and show overlapping composition (Fig. 6b) with more peraluminous leucogranites of Darbuk granite (Table 3). The garnet is not uncommon in the latter, and sometime its modal abundance can be $2-4 \%$. However, the LS and LG because of mineralogical control have very wide variation in their total REE. The REE patterns of LS and LG with or without garnet are shown in Fig. 7a along with OG, PG and CLG. The LS have more enriched REE abundance ( $\Sigma$ REE: 29-253; 103), while LG show $\Sigma$ REE ranging from 40-94 ppm with a mean value of around 55, but have enriched
HREE because of abundant garnet in them. The LS and LG show large variation in Eu anomaly from 0.51 to 1.37 and 0.15 to 0.77 respectively (Table 4). On the other tectonic discrimination diagrams LS behaves similar to LG as discussed above (Figs. 7b, 8-10). The foregoing discussion suggests that, the LS can be linked to leucogranites that form a complex melt flow intrusive sheet network.

\section{Discussion}

The main processes believed to give rise to crustal growth in modern plate tectonic settings is a two-stage melting process occurring mostly along convergent plate margins (e.g., Kay and Mahlburg-Kay, 1991). One involves the generation of large calc-alkaline Andeantype batholith, and the other produces evolved melts of average crustal composition by their remelting (e.g., Gromet and Silver, 1987). Most of the tectonic evolution of the crust in the Shyok-Darbuk section of SSZ can be explained by two-stage melting process involving the generation of orthogneiss, and temporal evolution towards increasingly more evolved felsic magmas during reworking processes.

\section{Petrogenesis of Orthogneiss (OG)}

Synchronous with the mid-Upper Cretaceous episode was the passive accretion of the Kohistan-Ladakh terrane to the Karakoram and closure of the SSZ (cf. Rex et al., 1988), leading to generation of syn- to post-accretion subduction related calc-alkaline magmas. These calc-alkaline Hbl-Bt orthogneiss of Shyok-Darbuk section have been reported to be of Early Eocene to Late-Cretaceous age, e.g., the $\mathrm{U}-\mathrm{Pb}$ zircon ages of 50-51 Ma for diorites from Tangtse and Darbuk (Ravikant et al., 2009).

The calc-alkaline I-type nature of OG from the Shyok-Darbuk section is evident from their tonalite to granodiorite composition (Fig. 5a and b), presence of euhedral to subhedral zoned oligoclase plagioclase (Fig. 4b), by the abundance of principal ferromagnesian minerals like rhombic to prismatic hornblende (Fig. 4a) and biotite, and OG having numerous enclaves of mafic rocks. The accessory minerals occurring as idioblastic to subidioblastic grains, e.g., the rhombic sphene (Fig. 4c), zircons and apatite also support for their I-type nature (cf. Chappell and White, 1974). The Nb, Ti, Sr and P provide an important clue in establishing emplacement environment (e.g., Tarney et al., 1981). The orthogneisses showing Nb, Ti and P troughs on the primitive mantle normalized diagram suggesting their derivation from arc source (Fig. 7b). The signature of syn-accretion subduction related arc environment of $\mathrm{OG}$ is evident from the tectonic discrimination diagrams (Figs. 8 and 10). The primitive to mature arc nature of the magma (Fig. 9) that produced the tonalitegranodiorites is also evident from low $\mathrm{Rb} / \mathrm{Sr}(\sim 0.10)$ and low $\mathrm{Nb}$ ( 14 ppm).

The observed geochemical features like, higher $\mathrm{CaO}, \mathrm{MgO}, \mathrm{Al}_{2} \mathrm{O}_{3}$, $\mathrm{Sr}$ and lower total alkalies, and fractionated REE pattern correlate the OG with late-orogenic granites defined by Harris et al. (1986). The late-orogenic granites were considered to be derived from the mafic/intermediate magmatic products of continental-margin subduction (Rogers and Greenberg, 1990). The LILE enrichment, high LILE/HFSE ratios, and often having large LREE/HREE ratios (e.g., high $\mathrm{La}_{\mathrm{N}} / \mathrm{Lu}_{\mathrm{N}}$ ratio of $\sim 37$ ) of OG (Table 4) may also suggest the presence of fluids during dehydration of subducting oceanic 
Table 4. Range and mean geochemical values of Orthogneiss, Porphyritic Granite, Coarse Leucogranite, Leucosome (migmatite zone) and Leucogranite along Shyok-Darbuk section, NE Ladakh, India

\begin{tabular}{|c|c|c|c|c|c|c|c|c|c|c|c|c|c|c|c|}
\hline & \multicolumn{3}{|c|}{ Hbl-Bi Orthogneiss (OG) } & \multicolumn{3}{|c|}{ Porphyritic Granite (PG) } & \multicolumn{3}{|c|}{ Coarse Leucogranite (CLG) } & \multicolumn{3}{|c|}{ Leucosome (LS) } & \multicolumn{3}{|c|}{ Leucogranite (LG) } \\
\hline & Min & Max & Mean & Min & Max & Mean & Min & Max & Mean & Min & Max & Mean & Min & Max & Mean \\
\hline $\mathrm{SiO}_{2}$ & 59.47 & 66.25 & 62.68 & 70.24 & 72.41 & 71.22 & 72.56 & 75.45 & 73.85 & 67.24 & 77.01 & 73.58 & 71.98 & 75.28 & 74.01 \\
\hline $\mathrm{Al}_{2} \mathrm{O}_{3}$ & 15.12 & 16.88 & 15.85 & 14.96 & 15.56 & 15.29 & 13.89 & 15.29 & 14.66 & 12.21 & 16.44 & 14.28 & 12.35 & 15.06 & 14.43 \\
\hline $\mathrm{TiO}_{2}$ & 0.58 & 0.90 & 0.80 & 0.15 & 0.43 & 0.30 & 0.03 & 0.16 & 0.07 & 0.04 & 0.65 & 0.17 & 0.00 & 0.29 & 0.06 \\
\hline $\mathrm{FeO}$ & 2.13 & 5.68 & 3.78 & 1.42 & 2.36 & 1.78 & 0.35 & 1.30 & 0.78 & 0.51 & 3.70 & 1.27 & 0.55 & 2.11 & 0.93 \\
\hline $\mathrm{MgO}$ & 2.92 & 4.57 & 4.14 & 0.40 & 0.92 & 0.59 & 0.06 & 0.41 & 0.27 & 0.14 & 1.51 & 0.39 & 0.09 & 0.48 & 0.18 \\
\hline $\mathrm{MnO}$ & 0.04 & 0.08 & 0.07 & 0.02 & 0.05 & 0.03 & 0.01 & 0.04 & 0.03 & 0.01 & 0.06 & 0.02 & 0.01 & 0.47 & 0.10 \\
\hline $\mathrm{CaO}$ & 3.02 & 4.82 & 4.27 & 1.44 & 2.48 & 2.01 & 1.31 & 2.18 & 1.63 & 1.11 & 3.65 & 1.74 & 0.77 & 1.71 & 1.39 \\
\hline $\mathrm{Na}_{2} \mathrm{O}$ & 4.20 & 5.27 & 4.79 & 4.42 & 5.14 & 4.72 & 3.66 & 4.56 & 4.10 & 3.12 & 5.42 & 3.98 & 2.96 & 5.27 & 4.27 \\
\hline $\mathrm{K}_{2} \mathrm{O}$ & 1.85 & 3.44 & 2.67 & 3.07 & 4.52 & 3.62 & 3.58 & 5.46 & 4.29 & 2.31 & 6.46 & 4.77 & 3.64 & 5.18 & 4.32 \\
\hline $\mathrm{P}_{2} \mathrm{O}_{5}$ & 0.24 & 0.46 & 0.40 & 0.02 & 0.17 & 0.11 & 0.01 & 0.13 & 0.06 & 0.00 & 0.29 & 0.05 & 0.00 & 0.08 & 0.02 \\
\hline $\mathrm{Ba}$ & 412 & 1364 & 714 & 544 & 1162 & 871 & 379 & 2188 & 681 & 260 & 3106 & 1078 & 13 & 967 & 449 \\
\hline V & 22 & 70 & 55 & 11 & 50 & 31 & 0 & 65 & 11 & 1 & 60 & 12 & 1 & 30 & 5 \\
\hline $\mathrm{Sc}$ & 5 & 13 & 9 & 3 & 5 & 4 & 3 & 8 & 5 & 2 & 6 & 4 & 3 & 6 & 4 \\
\hline $\mathrm{Ni}$ & 8 & 26 & 20 & 1 & 8 & 4 & 0 & 4 & 1 & 0 & 16 & 6 & 3 & 8 & 5 \\
\hline $\mathrm{Ga}$ & 15 & 23 & 18 & 19 & 27 & 23 & 13 & 25 & 21 & 13 & 21 & 17 & 19 & 26 & 21 \\
\hline $\mathrm{Pb}$ & 11 & 22 & 14 & 33 & 55 & 42 & 24 & 58 & 47 & 14 & 57 & 33 & 38 & 57 & 49 \\
\hline $\mathrm{Rb}$ & 60 & 118 & 83 & 120 & 241 & 192 & 91 & 236 & 175 & 59 & 144 & 110 & 158 & 257 & 197 \\
\hline $\mathrm{Sr}$ & 769 & 1064 & 856 & 259 & 691 & 480 & 171 & 625 & 274 & 201 & 710 & 441 & 41 & 305 & 196 \\
\hline Th & 6 & 44 & 11 & 8 & 42 & 23 & 4 & 25 & 10 & 1 & 50 & 14 & 6 & 28 & 12 \\
\hline $\mathrm{U}$ & 1 & 4 & 2 & 4 & 6 & 5 & 4 & 7 & 6 & 3 & 6 & 4 & 4 & 13 & 7 \\
\hline $\mathrm{Y}$ & 11 & 24 & 15 & 6 & 10 & 7 & 5 & 13 & 9 & 3 & 31 & 11 & 8 & 66 & 22 \\
\hline $\mathrm{Zr}$ & 179 & 234 & 206 & 52 & 168 & 131 & 34 & 151 & 53 & 19 & 347 & 121 & 30 & 145 & 53 \\
\hline $\mathrm{Nb}$ & 7 & 27 & 14 & 3 & 15 & 8 & 2 & 19 & 8 & 1 & 26 & 8 & 4 & 20 & 11 \\
\hline $\mathrm{La}$ & 50.98 & 58.80 & 55.17 & 10.94 & 75.73 & 38.79 & 12.65 & 21.78 & 16.81 & 5.55 & 63.53 & 24.57 & 5.27 & 12.79 & 9.27 \\
\hline $\mathrm{Ce}$ & 91.41 & 111.81 & 98.12 & 21.30 & 144.95 & 74.97 & 26.08 & 45.49 & 34.96 & 10.83 & 114.34 & 45.22 & 11.25 & 25.52 & 19.01 \\
\hline $\operatorname{Pr}$ & 9.37 & 12.05 & 10.59 & 2.15 & 15.57 & 7.74 & 2.81 & 5.04 & 3.84 & 1.13 & 11.42 & 4.67 & 1.30 & 3.08 & 2.12 \\
\hline $\mathrm{Nd}$ & 36.19 & 47.70 & 39.74 & 8.11 & 60.37 & 28.92 & 11.10 & 19.95 & 15.01 & 4.46 & 40.64 & 16.72 & 5.39 & 13.62 & 8.62 \\
\hline Sm & 5.09 & 6.59 & 5.75 & 1.64 & 8.96 & 4.47 & 2.20 & 3.82 & 3.16 & 0.97 & 6.14 & 2.91 & 1.63 & 4.47 & 2.38 \\
\hline $\mathrm{Eu}$ & 1.40 & 1.65 & 1.52 & 0.57 & 1.61 & 0.89 & 0.64 & 0.93 & 0.76 & 0.23 & 1.35 & 0.68 & 0.22 & 0.41 & 0.30 \\
\hline $\mathrm{Gd}$ & 4.48 & 5.26 & 4.92 & 1.45 & 7.90 & 3.60 & 1.57 & 3.25 & 2.60 & 0.91 & 5.55 & 2.64 & 1.51 & 4.40 & 2.14 \\
\hline $\mathrm{Tb}$ & 0.54 & 0.70 & 0.63 & 0.21 & 0.99 & 0.43 & 0.17 & 0.43 & 0.33 & 0.09 & 0.79 & 0.36 & 0.24 & 1.12 & 0.46 \\
\hline Dy & 2.34 & 3.80 & 3.23 & 1.15 & 4.99 & 1.97 & 0.69 & 2.01 & 1.54 & 0.38 & 4.45 & 2.04 & 1.45 & 10.07 & 3.67 \\
\hline Ho & 0.33 & 0.65 & 0.55 & 0.15 & 0.84 & 0.30 & 0.09 & 0.29 & 0.22 & 0.06 & 0.92 & 0.38 & 0.27 & 2.35 & 0.82 \\
\hline $\mathrm{Er}$ & 0.95 & 1.82 & 1.51 & 0.46 & 2.33 & 0.84 & 0.23 & 0.75 & 0.56 & 0.17 & 2.49 & 1.06 & 0.77 & 7.70 & 2.61 \\
\hline $\mathrm{Tm}$ & 0.11 & 0.26 & 0.21 & 0.04 & 0.32 & 0.11 & 0.03 & 0.12 & 0.07 & 0.02 & 0.37 & 0.16 & 0.12 & 1.51 & 0.46 \\
\hline $\mathrm{Yb}$ & 0.59 & 1.60 & 1.29 & 0.26 & 1.83 & 0.64 & 0.16 & 0.74 & 0.44 & 0.10 & 2.49 & 0.94 & 0.70 & 11.08 & 3.04 \\
\hline $\mathrm{Lu}$ & 0.08 & 0.23 & 0.18 & 0.04 & 0.25 & 0.09 & 0.03 & 0.10 & 0.06 & 0.02 & 0.37 & 0.14 & 0.12 & 1.97 & 0.51 \\
\hline$\Sigma$ REE & 206.67 & 248.38 & 223.40 & 48.943 & 26.63 & 163.74 & 58.44 & 103.28 & 80.38 & 29.22 & 252.62 & 102.48 & 39.96 & 94.20 & 55.41 \\
\hline mol.A/CNK & 0.91 & 0.99 & 0.95 & 0.98 & 1.06 & 1.02 & 0.93 & 1.05 & 1.00 & 0.83 & 1.04 & 0.95 & 0.96 & 1.04 & 1.01 \\
\hline mg\# & 64 & 71 & 67 & 33 & 46 & 37 & 12 & 51 & 38 & 25 & 48 & 35 & 16 & 33 & 26 \\
\hline $\mathrm{Na}_{2} \mathrm{O} / \mathrm{K}_{2} \mathrm{O}$ & 1.4 & 2.8 & 1.9 & 1.0 & 1.6 & 1.3 & 0.7 & 1.2 & 1.0 & 0.5 & 2.3 & 0.9 & 0.7 & 1.4 & 1.0 \\
\hline $\mathrm{Rb} / \mathrm{Sr}$ & 0.08 & 0.15 & 0.10 & 0.17 & 0.92 & 0.48 & 0.15 & 1.21 & 0.77 & 0.08 & 0.65 & 0.31 & 0.57 & 2.65 & 1.22 \\
\hline $\mathrm{La} / \mathrm{Nb}$ & 2.0 & 8.4 & 4.7 & 2.6 & 10.8 & 6.9 & 1.0 & 5.9 & 2.8 & 1.2 & 14.1 & 6.4 & 0.6 & 1.1 & 0.8 \\
\hline $\mathrm{Ba} / \mathrm{La}$ & 9 & 23 & 14 & 21 & 27 & 25 & 28 & 119 & 52 & 15 & 157 & 56 & 1 & 73 & 41 \\
\hline $\mathrm{K} / \mathrm{Zr}$ & 66 & 148 & 109 & 152 & 508 & 257 & 228 & 1059 & 744 & 73 & 2762 & 611 & 231 & 1239 & 786 \\
\hline $\mathrm{K} / \mathrm{Y}$ & 1036 & 2708 & 1570 & 2687 & 8089 & 4700 & 2588 & 8258 & 4116 & 821 & 15476 & 4926 & 574 & 3872 & 2510 \\
\hline$(\mathrm{La} / \mathrm{Sm})_{\mathrm{N}}$ & 5.51 & 6.44 & 5.95 & 3.94 & 6.31 & 5.55 & 2.89 & 3.61 & 3.29 & 1.49 & 4.60 & 2.89 & 1.38 & 2.65 & 1.97 \\
\hline$(\mathrm{Gd} / \mathrm{Lu})_{\mathrm{N}}$ & 2.78 & 7.62 & 3.85 & 5.04 & 11.06 & 7.96 & 3.11 & 7.88 & 5.76 & 0.19 & 17.28 & 4.62 & 0.23 & 1.64 & 0.78 \\
\hline$(\mathrm{La} / \mathrm{Lu})_{\mathrm{N}}$ & 24.88 & 77.60 & 36.97 & 42.10 & 123.52 & 82.36 & 13.90 & 47.39 & 32.79 & 0.01 & 0.28 & 0.09 & 0.03 & 2.67 & 0.86 \\
\hline Eu anomaly & 0.84 & 0.90 & 0.88 & 0.53 & 0.79 & 0.68 & 0.72 & 1.07 & 0.84 & 0.51 & 1.37 & 0.84 & 0.15 & 0.77 & 0.47 \\
\hline
\end{tabular}

lithosphere. This requires partial melting of a precursor source of a quartz eclogites or eclogite source (e.g., Buma et al., 1971; Jahn et al., 1980; Ewing, 1979). Alternatively, the Y-depleted and fractionated HREE trend are also consistent with hydrous partial melting of an amphibole and/or garnet bearing mafic source (cf. Sheraton et al. 1985). Further, the linear trends of decreasing $\mathrm{Zr}, \mathrm{TiO}_{2}$, and $\mathrm{P}_{2} \mathrm{O}_{5}$ against $\mathrm{SiO}_{2}$ (Fig. 6a) reflect the fractional crystallization of zircon, titanite and apatite. The REE of these rocks also show fractionated trends, with small or no Eu anomaly. The crystallization of sphene has a control on their LREE fractionation trend, while crystallization of hornblende can be attributed to the cause of HREE depletion without changing Eu anomaly (cf. Frey et al., 1978), and the large concentrations of $\mathrm{Sr}$ and $\mathrm{Ba}$ explains for no significant $\mathrm{Eu}$ anomalies.

\section{Petrogenesis of Late Phase Granitoids (PG, CLG and $L G$ )}

In the Karakoram extremely large amounts of crustal shortening, thickening, metamorphism and magmatism followed the India-Asia collision (cf. Searle, 1991). The ending of I-type, subduction-related granite injection of the Meso-Cainozoic orogenic evolution of the Himalaya-Transhimalaya-Karakoram realm spanning from midCretaceous to Eocene time was followed by intracontinental subduction after closure of the oceanic realm. The latter is limited to a small volume of very uniform anatectic granite produced during a 10-15 Ma period (Srimal et al., 1987). In Shyok-Darbuk section of Pangong Range too one can see, an early phase of crustal growth in 

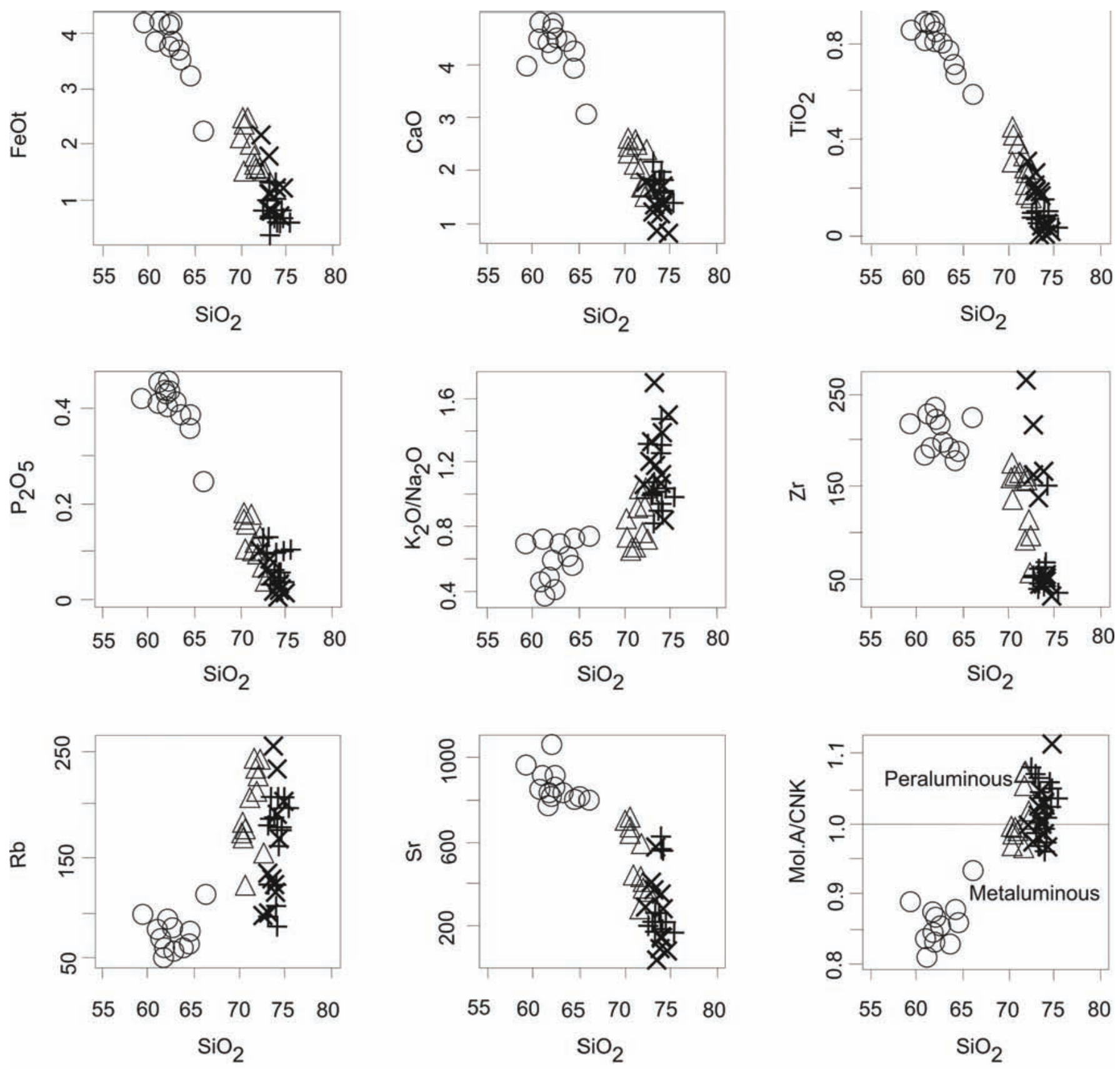

Figure 6a. Harker's variation diagram of some major and trace elements. Symbols as in Fig. 5.

the form of subduction-related Hbl-Bt orthogneisses (OG), followed by a late phase younger Miocene collision-related granites (PG, CLG and LG). The latter are mostly crustally derived two mica \pm garnet leucogranites and Bt-leucogranites (including pegmatitic dykes), and have crystallization ages between 19.1 $\pm 1.1 \mathrm{Ma}$ (Ravikant et al., 2009) and 13.7 $\pm 0.2 \mathrm{Ma}$ (Phillips et al., 2004). The exhumation of the Pangong migmatites and leucogranites between 18 and 11 Ma has also been suggested by Searle et al. (1998).

The post-collision magmatism in the Shyok-Darbuk section occur in the form of porphyritic granites (PG), coarse leucogranites (CLG), leucogranites (LG) and associated pegmatites in the host orthogneiss and metapelites. These different phases of granitoids viz. the PG, CLG and LG, though show a distinctive identity in the field with cross cutting relationships (Figs. 3h-j), geochemically, they define common trends with overlapping range of $\mathrm{SiO}_{2}$ in the range of 70-75 wt. \%, supporting their derivation from a common parent source (Table 4). They compositionally range from granodiorite to granite, and are metaluminous to peraluminous. The petrochemical signatures of rocks can best be explained through re-melting of earlier calc-alkaline orthogneiss and metapelites. The genetic link between late phase granitoids and the host orthogneiss is observed from the geochemical coherence of these rocks on inter-element diagrams and Harker's variation diagram (Fig. 6a). The partial melting of orthogneiss has resulted in enriched incompatible elements and depletion of compatible elements, and total REE of late phase granitoids (Fig. 7). However, the involvement of subduction nature and plotting in the mature continental arc field of the late phase granitoids reflect their precursor signatures (Figs. 8-10). The slight peraluminous nature, enriched LILE and LREE elements of the late phase granitoids also points out to some involvement of sedimentary source material. An 

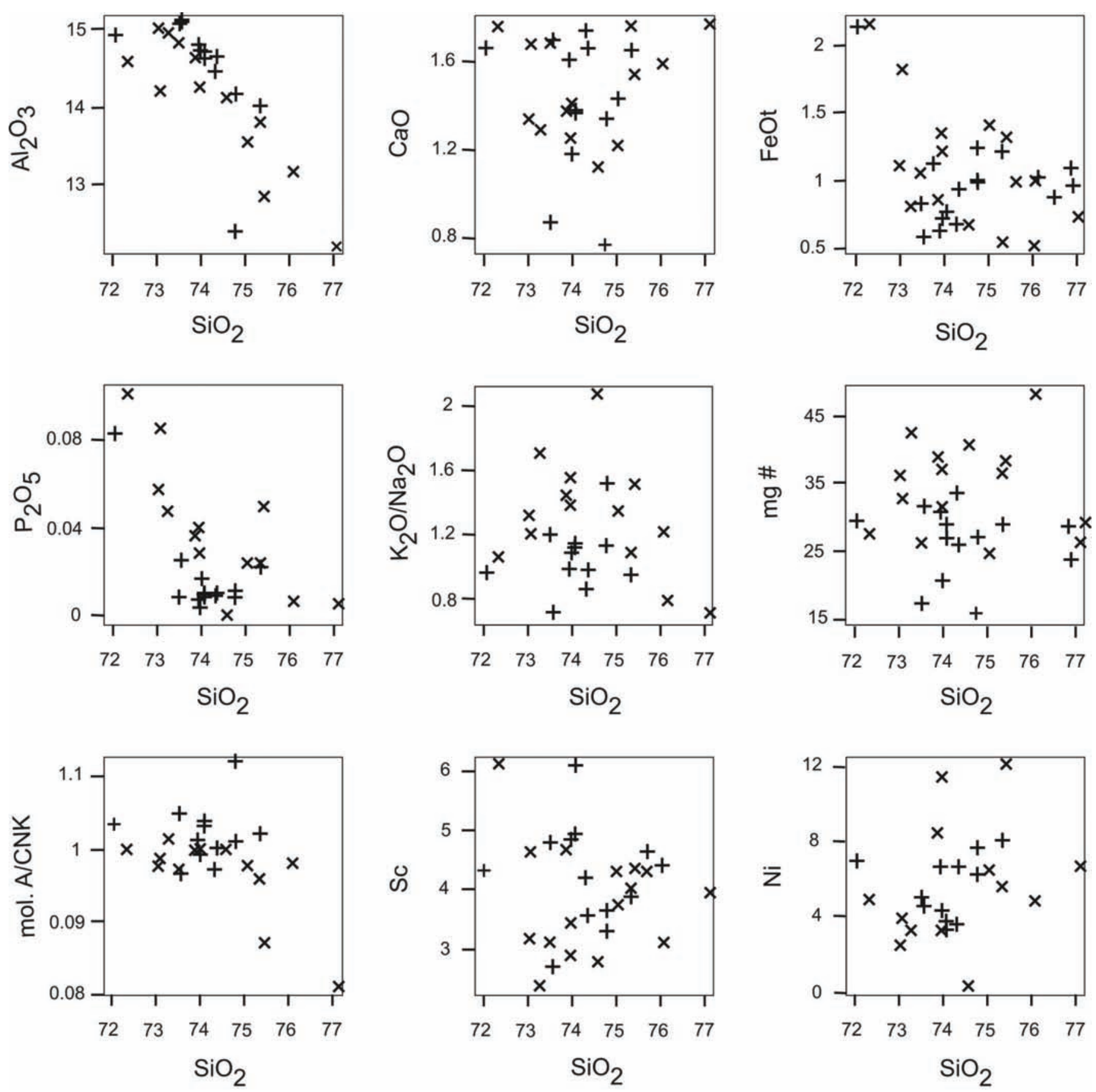

Figure 6b. Plot showing overlapping composition of some major and trace elements shown by Leucosome (LS) from migmatites - 'cross', and Leucogranite (LG) from Darbuk Granite - 'plus'.

early Tertiary crustal thickening and regional metamorphism provided the heat for melting that produce the late phase leucogranites. The metamorphism of metapelites hosting granites in Shyok-Darbuk section show pressure-temperature estimates of $\sim 6.2 \mathrm{kbars}$ and $670^{\circ} \mathrm{C}$ respectively (Rameshwar Rao and Rai, 2003), which is ideal to generate anatectic melts in the region. Thus, the remelting, interaction and mixing of magmas derived from asthenospheric mantle wedge (orthogneiss) and crustal (metapelite) sources producing the younger leucogranites seems to have played fundamental role for the crustal growth and temporal evolution towards increasingly more evolved felsic magmas. Further, the absence of hornblende as one of the mafic minerals in late phase granitoids may also suggest for low degrees of partial melting. Figure 6a also suggests that the melts underwent fractional crystallization with CLG and LG showing greater fractionation than $\mathrm{PG}$.

The petrogenetic modeling, irrespective of whether the protolith is granitic, granodioritic, tonalite, metapelites or metapsammites, the amount of melt generated in the absence of fluids is few percent involving high temperatures exceeding $800^{\circ} \mathrm{C}$. However, water-flux induced melting of protoliths occurs at low temperatures of $\sim 700^{\circ} \mathrm{C}$, and can result in large volumes of granitic melts (cf. Sawyer, 2010, and the references therein). The $\mathrm{Rb} / \mathrm{Sr}$ ratios have been used as a discriminator of water-fluxed vs dehydration melting. The $\mathrm{Rb} / \mathrm{Sr}$ ratio of LS from migmatites is $\sim 0.31$ and for LG from Darbuk granite 

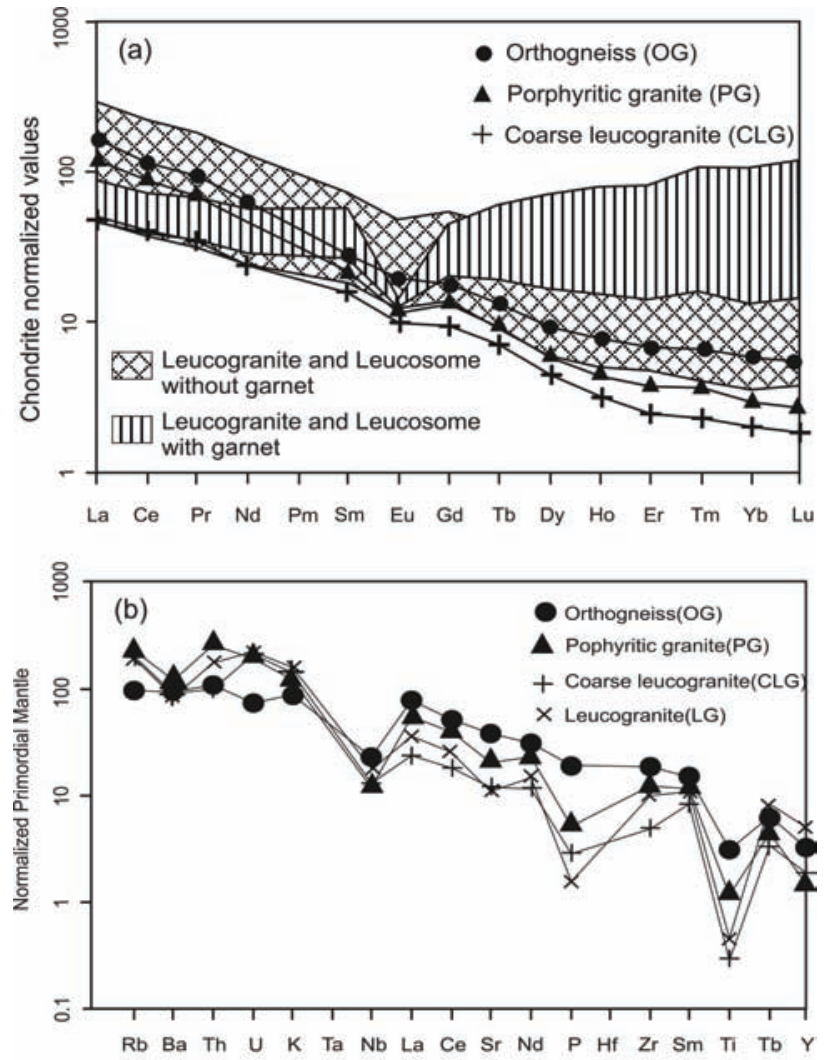

Figure 7. (a) showing Chondrite normalized REE patterns of the mean values of $O G, P G, C L G$, along with the distribution pattern of $L S$ and $L G$, and (b) showing the primordial mantle normalized patterns (after Wood et al., 1979) of the mean values of $O G, P G$, $C L G$ and $L G$.

is $\sim 1.22$ (Tables 4), which at face value is consistent with a low Rb$\mathrm{Sr}$ fractionation during water-flux induced melting (cf. Harris and Inger, 1992; Harris et al., 1995). Further, Acosta-Vigil et al. (2006) also showed that in water-saturated partial melting of granite the melt phase occupied all the grain boundaries and triple points in the rock. The LS show a range in quartz morphologies, from high-aspect ratio films to isolate rounded to irregular grains, as the microstructure

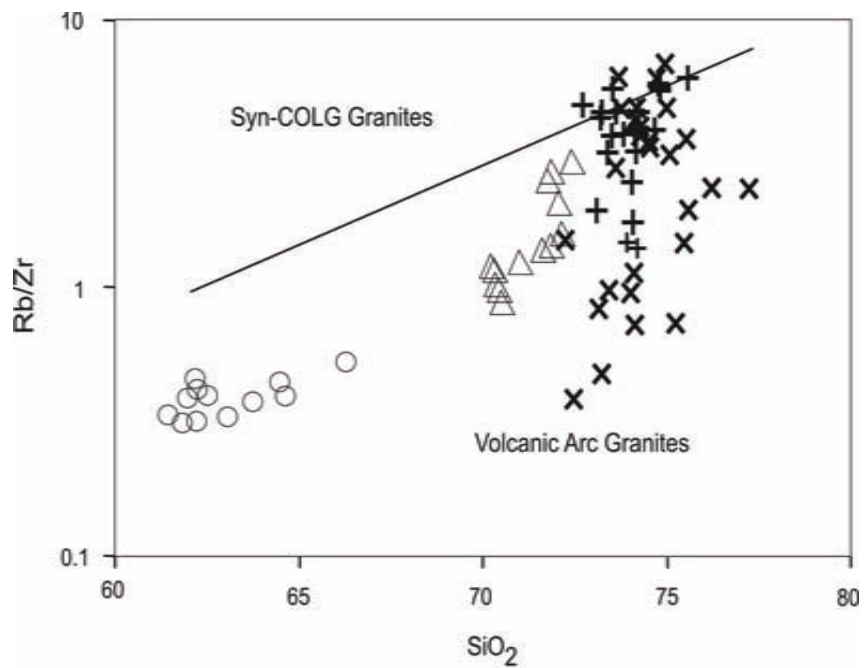

Figure 8. The plotting of rocks on the tectonic discrimination diagram of $\mathrm{SiO}_{2}$ vs $\mathrm{Rb} / \mathrm{Zr}$, fields after Harris et al., 1986. Symbols as in Fig. 5.

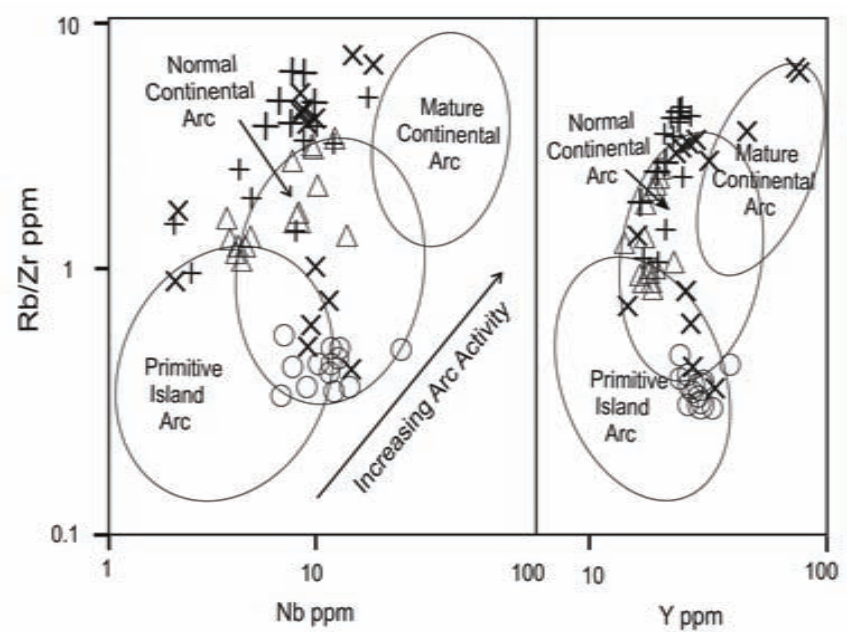

Figure 9. The plotting of rocks on the tectonic variation diagrams of Nb ppm and Y ppm vs Rb/Zr, fields after Brown et al. (1984). Symbols as in Fig. 5.

progressively equilibrated after crystallization. Also, the microstructure in residual rocks show straight grain boundaries in biotite without reaction products, but plagioclase, quartz and microcline all have irregular grain boundaries due to dissolution (Fig. 4a and c), suggesting for the involvement of water-flux during anatexis. The equations involved in the partial melting could include, $\mathrm{Kfs}+\mathrm{Qtz}+\mathrm{Pl}+\mathrm{H}_{2} \mathrm{O}=$ melt, and/or those involving quartz, plagioclase and biotite (cf. Gardien et al., 2000), such as $\mathrm{Pl}^{1}+\mathrm{Qtz}+\mathrm{Bt}+\mathrm{H}_{2} \mathrm{O}=$ melt $+\mathrm{Amph}+\mathrm{Pl}^{2} \pm \mathrm{Ttn}$. The latter equation according to Slagstad et al. (2005) occurs at temperatures between 700 and $720^{\circ} \mathrm{C}$. They also further suggested that, the shear zones are widely thought to be the pathways, along which the aqueous fluids that caused anatexis were migrated.

Some of the recent studies carried out in the Pangong Range suggest that the granodiorites from the Tangtse gorge (74 Ma) and granite around Muglib (63 and $56 \mathrm{Ma}$ ) show geochronological and isotopic characteristics to those of the Ladakh batholith (cf. Ravikant et al. 2009), while the leucosomes of Pangong Metamorphic Complex rooting in different source rocks merge to form a hierarchy of magma channels, feeding into stocks, plutons and ultimately into the

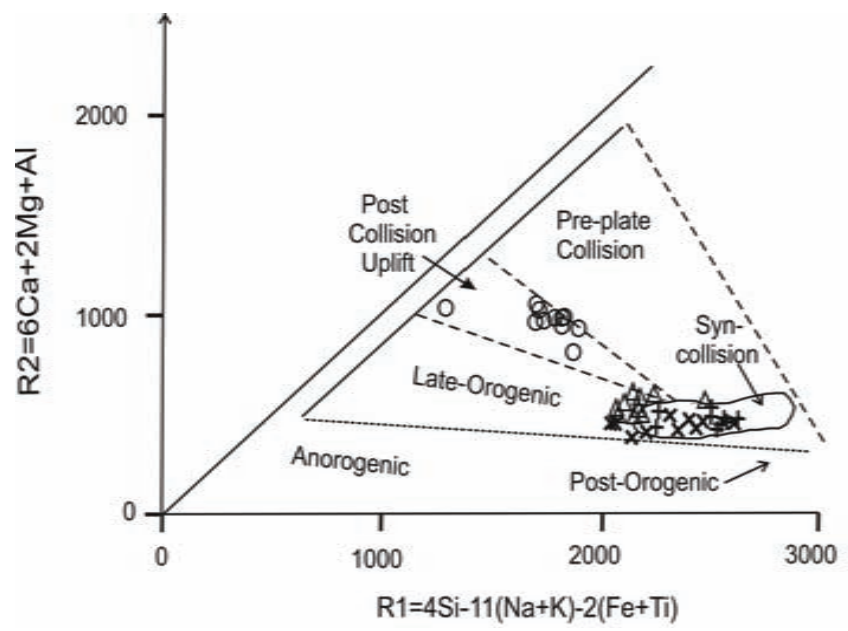

Figure 10. The plotting of rocks on R1-R2 tectonic discrimination diagram, fields after Batchelor and Bowden (1985). Symbols as in Fig.5. 

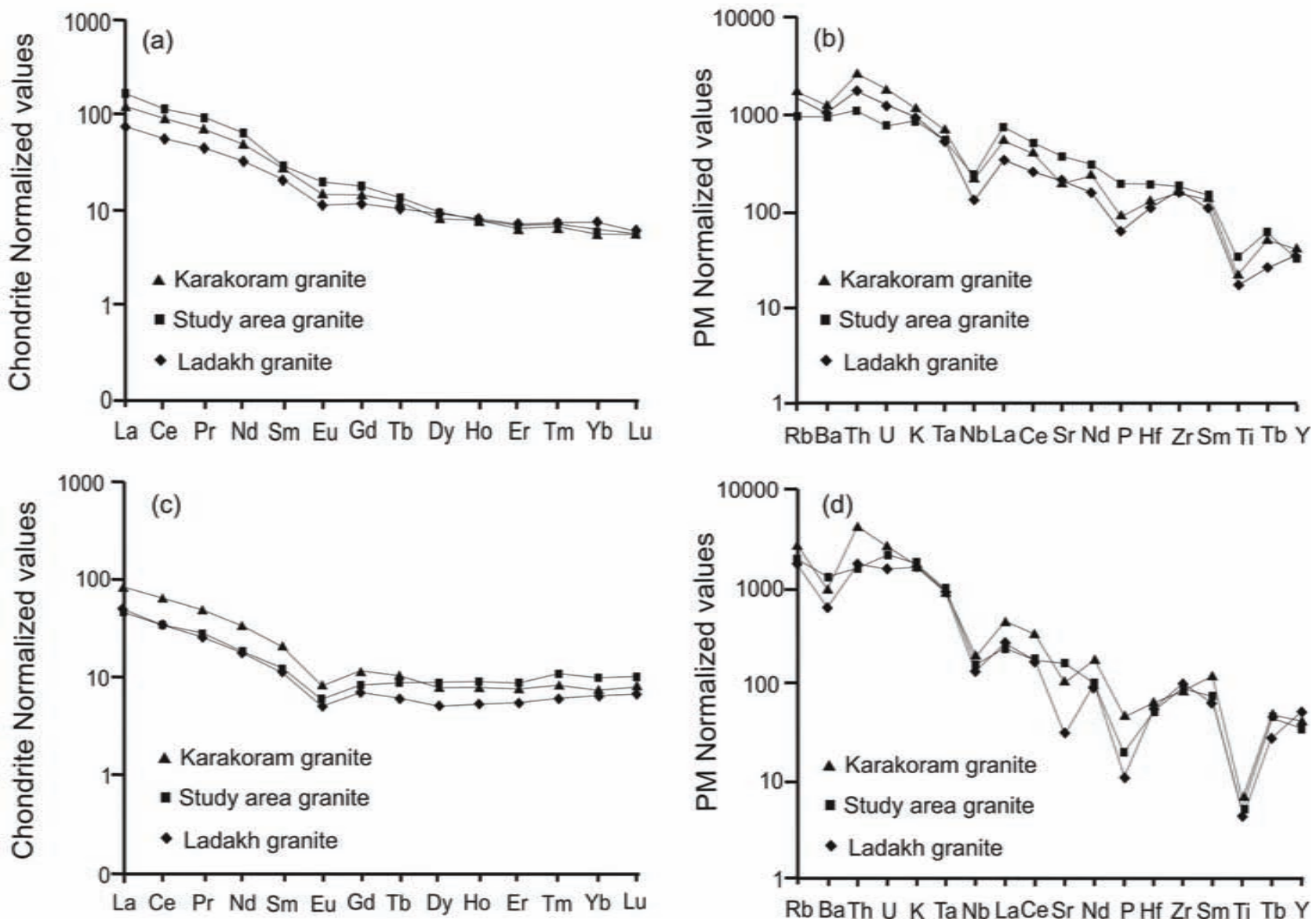

Figure 11. (a) REE and (b) Primordial Mantle patterns of OG compared with Ladakh and Karakoram granitoids. (c) REE and (d) Primordial Mantle patterns of LG compared with Ladakh and Karakoram granitoids. Source of the data is from Crawford and Searle (1992); Guillot and Le Fort (1995); Ahmed et al. (1998); Upadhayay et al. (2005); Rai and Rameshwar Rao (2009); and Rameshwar Rao and Rai (2009a, b).

Karakoram Batholith (cf. Reichardt et al., 2010). However, the comparison of the geochemical data of OG and LG from the present study with the Ladakh and Karakoram granitoids (Fig. 11) does not reveal such picture. In present study, the normalized REE and Primordial Mantle values of Ladakh and Karakoram granodiorites and leucogranites are compared (Fig. 11) respectively with OG and LG (Tables 1 and 4). The source data for Ladakh and Karakoram granitoids is given in figure captions. It has been observed that the REE pattern of OG is more close to the REE pattern of Karakoram Granodiorites (Fig. 11a), and similar observation is observed for compatible elements (Fig. 11b). The LG on the other hand have LREE closely matching with Ladakh leucogranites, while HREE are comparable with Karakoram leucogranites (Fig. 11c). There is also no clear correlation of compatible and incompatible elements (Fig. 11d). So, from the present study, no conclusive relation can be drawn, though at the same time the comparisons made in recent times seems to be a distant reality, and require more isotopic and geochemical data to come out at such conclusions.

\section{Conclusions}

It is conceivable that in the Shyok-Darbuk corridor of Pangong range, the examples mentioned, two groups of independently generated magmas were produced at different time framework. The crustal evolution in what is now ostensibly a single complex is through the generation of orthogneiss followed by generation of increasingly more evolved felsic magmas. Each type is indicative of the origin, evolution, and geodynamic environment.

In the light of the geochemical evidence the conclusions of the present study is summarized as follows:

1 The arc formation and accretion were the most important geological processes during Late Cretaceous to Eocene times and dominated the evolution of Shyok orthogneiss, which have originated from the mafic source in arc setting.

2 The crustal evolution in changing geodynamic settings i.e., from arc environments to continental collision causing thickening and melting of mid to lower crust resulted in the addition of enriched anatectic melts to the accreted arc terrane in Darbuk region.

3 The generation of these felsic melts during post-collision Miocene times had played a fundamental role in the evolution ShyokDarbuk terrane in the form of PG, CLG, LG, and associated pegmatites, along with the generation of leucosomes in the migmatites.

4 More so, the correlation of the OG and LG with Ladakh and Karakoram granitoids does not provide any conclusive relation with either, and is still a matter of debate, but of importance for palaeoplate reconstructions. 


\section{Acknowledgements}

We are grateful to Director, Wadia Institute of Himalayan Geology, for providing facilities and giving permission to publish this paper. We thank Dr. N.K. Saini, Mr. Chandra Shekhar and Dr. P.P. Khanna for their help in generating the geochemical data on XRF and ICPMS instruments. We are also thankful to the two anonymous reviewers for providing valuable suggestions and comments which improved the manuscript significantly.

\section{References}

Acosta-Vigil, A., London, D., and Morgan, G.B., 2006, Experiments on the kinetics of partial melting of a leucogranite at $200 \mathrm{MPa}$ and $690-800^{\circ} \mathrm{C}$ : compositional variability of melts during the onset of $\mathrm{H}_{2} \mathrm{O}$-saturated crustal anatexis: Contributions to Mineralogy and Petrology, v. 151, pp. 539-557.

Ahmad, T., Thakur, V.C., Islam, R., Khanna, P.P., and Mukherjee, P.K., 1998, Geochemistry and geodynamics implications of magmatic rocks from the Trans-Himalayan arc: Geochemical Journal, v. 32, pp. 383-404.

Andersson, U.B., 1991, Granitoid episodes and mafic/felsic magma interaction in the Svecofennian of the Fennoscandian Shield, with main emphasis on the approximately 1.8 Ga plutonics: Precambrian Research, v. 51 (14), pp. 127.149.

Barker, F., and Arth, J.G., 1976, Generation of trondhjemite-tonalitic liquids and Archean bimodal trondhjemite-baslat suites: Geology, v. 4, pp. 596600 .

Batchelor, R.A., Bowden, P., 1985, Petrogenetic interpretation of granitoid rock series using multicationic parameters: Chemical Geology, v. 48, pp. 43-55.

Brown, G.C., Thorpe, R.S., and Webb, P.C., 1984, The geochemical characteristics of granitoids arcs and comments on magma sources: Journal of Geological Society of London, v. 141, pp. 413-426.

Buma, G., Frey, F.A., and Wones, D.R., 1971, New England granites: trace element evidence regarding their origin and differentiation: Contribution to Mineralogy and Petrology, v. 31, pp. 300-320.

Castro, A., Moreno-Ventas, I., and Dela Rosa, J.D., 1991, H-type (hybrid) granitoids: a proposed revision of the granite-type classification and nomenclature: Earth Science Review, v. 31, pp. 237-253.

Chappell, B. W., and White, A J R., 1974, Two contrasting granite types: Pacific Geology, v. 8, pp. 173-174.

Choudhary, B.K., 1983, Structure, metamorphism and deformation history of Pangong Tso area, eastern Ladakh, in Thakur, V.C., and Sharma, K.K., eds, Geology of Indus Suture Zone of Ladakh, WIHG, Dehradun, pp. 107-116.

Clift, P.D., Hannigan, R., Blusztajn, J.S., and Drant, A.E., 2002, Geochemical evolution of the Dras-Kohistan Arc during collision with Eurasia: evidence from the Ladakh Himalaya, India: The Island Arc, v. 11 (4), pp. 255-264.

Crawford, M.B., and Searle, M.P., 1992, Field relationships and geochemistry of pre-collisional (India-Asia) granitoid magmatism in the central Karakoram, northern Pakistan: Tectonophysics, v. 206, pp. 171-192.

Debon, F., Le Fort, P., Dautel, D., Sonet, J., and Zimmermann, J.L., 1987, Granitoids of western Karakoram and northern Kohistan (Pakistan): a composite Mid-Cretaceous to Upper Cenozoic magmatism: Lithos, v. 20, pp. 19-24.

Debon, F., Le Fort, P., and Sonet, J., 1981, Granitoid belts west and south of Tibet: about their geochemical trends and $\mathrm{Rb}-\mathrm{Sr}$ isotopic studies, in Proceedings of Symposium on Qinghai-Xizang Plateau, Geological and ecological studies of Qinghai-Xizang Plateau, Beijing, Science Press, pp. 395-405.

Ewing, T.E. 1979, Two calc-alkaline volcanic trends in the Archean: trace element evidence: Contribution to Mineralogy and Petrology, v. 71, pp. 1-7.
Frey, F.A., Chappell, B.W., and Roy, S.D., 1978, Fractionation of rare earth elements in the Tuolumne Intrusive Series, Sierra Nevada batholith, California: Geology, v. 6, pp. 239-242.

Gardien, V., Thompson, A.B., and Ulmer, P., 2000, Melting of biotite+plagioclase+quartz gneisses: the role of $\mathrm{H}_{2} \mathrm{O}$ in the stability of amphibole: Journal of Petrology, v. 41, pp. 651-666.

Gray, C. M., 1984, An isotopic mixing model for the origin of granitic rocks in southeastern Australia: Earth and Planetary Science Letters, v. 70, pp. $47-60$.

Gromet, L.P., and Silver, L.T., 1987, REE variation across the Peninsular range Batholith: implication for batholitic petrogenesis and crustal growth in magmatic arcs: Journal of Petrology, v. 28, pp. 75-125.

Guillot, S., and Le Fort, P., 1995, Geochemical constraints on the bimodal origin of High Himalayan leucogranites: Lithos, v. 35 (3-4), pp. 221234.

Harpum, J.R., 1963, Petrographic classification of granitic rocks in Tanganyika by partial chemical analyses: Records Geological Survey Tanganyika, v. 10, pp. 80-88.

Harris N, Ayres M, and Massey J., 1995, Geochemistry of granitic melts produced during the incongruent melting of muscovite: implications for the extraction of Himalayan leucogranite magmas: Journal of Geophysical Research, v. 100, pp. 15767-15777.

Harris, N., and Inger, N B W, 1992, Trace elements modeling of pelite-derived granites: Contribution Mineralogy Petrology, v. 110, pp. 46-56.

Harris, N.B.W., Pearce, J.A., and Tindle, A.G., 1986, Geochemical characteristics of collision zone magmatism, in Coward, M.P., and Ries, A.C., eds, Collision Tectonics, Geological Society Special Publication, v. 19 , pp. $67-81$

Hawkesworth, C.J., and Vollmer, R., 1979, Crustal contamination versus enriched mantle: ${ }^{143} \mathrm{Nd} /{ }^{144} \mathrm{Nd}$ and ${ }^{87} \mathrm{Sr} /{ }^{86} \mathrm{Sr}$ evidence from the Italian volcanics: Contributions to Mineralogy and Petrology, v. 69(2), pp. 151.165 .

Jahn, B., Auvray, B., Blais, S., Capdevila, R., Cornichet, J., Vidal, R., and Hameurt, J., 1980, Trace element geochemistry and petrogenesis of Finnish greenstone belts: Journal of Petrology, v. 21, pp. 201-244.

Jain, A.K., and Singh, S., 2008, Tectonics of the southern Asian plate margin along the Karakoram Shear Zone: constrains from field observations and U-Pb SHRIMP ages: Tectonophysics, v. 451, pp. 186-205.

Kay, R. W., and Mahlburg-Kay, S., 1991, Creation and destruction of lower continental crust: Geological Rundsch, v. 80, pp. 259-278.

Khanna, P.P., Saini, N.K., Mukherjee, P.K., and Purohit, K.K., 2009, An appraisal of ICP-MS technique for determination of REEs: long term QC assessment of Silicate Rock Analysis: Himalayan Geology, v. 30, pp. 95-99.

Kretz, R., 1983, Symbols for rock forming minerals: American Mineralogist, v. 34 , pp. $345-368$.

Kumar, S., 2010, Mafic to hybrid microgranular enclaves in the Ladakh Batholith, Northwest Himalaya: Implication on Calc-alkaline Magma Chamber Processes: Journal of the Geological Society of India: v. 76, pp. $5-25$

Kumar, S., and Singh, B., 2008, Mineralogy and Geochemistry of mafic to hybrid microgranular enclaves and felsic host of Ladakh batholith, Northwest Himalaya: Evidence of multistage complex magmatic processes: Himalayan Journal of Sciences, vol. 5, Issue 7 (Special Issue), pp. 130-131

Le Fort, P., 1986, Metamorphism and magmatism during the Himalayan Collision, in: Coward, M.P., and Ries, A.C., eds, Collision Tectonics, Geological Society Special Publication, v. 19, pp. 159-172.

Petterson, M. G., and Windley, B.F., 1985, Rb-Sr dating of the Kohistan arcbatholith in the Trans-Himalaya of north Pakistan, and tectonic implications: Earth and Planetary Science Letters, v. 74, pp. 45-57.

Phillips, R. J., 2008, Geological map of the Karakoram fault zone, Eastern Karakoram, Ladakh, NW Himalaya: Journal of Maps, pp. 21-37.

Phillips, R.J., Parrish, R.R., and Searle, M.P., 2004, Age constraints on ductile deformation and long-term slip rates along the Karakoram fault zone, Ladakh: Earth and Planetary Science Letters, v. 226, pp. 305-319. 
Rai, H., 1986, Some geological observations on the area between Khalsar and Darbuk in the Shyok valley, Ladakh: Journal of Geological Society of India, v. 27, pp. 512-516.

Rai, H., 1997, Geology of the Shyok Tectonic Zone in eastern Ladakh, India: Journal of Geological Society of India, v. 49, pp. 727-730.

Rai, H., 2008, Petrogenesis of Shyok volcanics, Ladakh Trans-Himalaya, India: Memoir Geological Society of India, v. 72, pp. 47-72.

Rai, H., and Rameshwar Rao, D., 2005, Geochemical and isotopic studies of the granitoids exposed on the southern slope in the Sasoma-Saser Brangra Section of Karakoram batholith, Jammu and Kashmir: Journal Geological Society of India, v. 65, pp. 325-334.

Rai, H., and Rameshwar Rao, D., 2009, Granite magmatism along southern margin of eastern Karakoram, Ladakh, India, in Kumar, S, ed., Magmatism, Tectonism and Mineralization, Macmillan Publishers, pp. 102-116.

Rameshwar Rao, D., and Rai, H., 2003, Geochemical and geochronological studies of the Darbuk granite, and studies of associated metamorphic rocks from the Darbuk-Tangtse regions of Ladakh district, India: Indian Journal of Geochemistry, v. 18, pp. 55-76.

Rameshwar Rao, D., and Rai, H., 2009a, Geochemistry of granitoids from the Shyok tectonic zone near Tangtse, Ladakh, India: Himalayan Geology, v. 30, pp. 35-44.

Rameshwar Rao, D., and Rai, H., 2009b, Geochemical studies of granitoids from Shyok tectonic zone of Khardung-Panamik section, Ladakh, India: Journal Geological Society of India, v. 74, pp. 363-374.

Ravikant, V., 2006, Utility of Rb-Sr geochronology in constraining Miocene and Cretaceous events in the eastern Karakoram, Ladakh, India: Journal of Asian Earth Sciences, v.27, no.4, pp. 534-543.

Ravikant, V., Wu, F.-Y., and Ji, W.-Q., 2009, Zircon U-Pb and Hf isotopic constraints on petrogenesis of the Cretaceous-Tertiary granites in eastern Karakoram and Ladakh, India: Lithos, v. 110 (1-4), pp.153-166.

Reichardt, H., Weinberg, R.F., Andersson, U.B., and Fanning, C.M., 2010, Hybridization of granitic magmas in the source: The origin of the Karakoram Batholith, Ladakh, NW India: Lithos, v. 116, pp. 249-272, doi:10.1016/j.lithos.2009.11.013

Rex, A.J., Searle, M.P., Tirrul, R., Crawford, M.B., Prior, D.J., Rex, D.C., and Barnicoat, A.C., 1988, The geochemical and tectonic evolution of the central Karakoram, North Pakistan: Philosophical Transactions of the Royal Society of London, Series A: Mathematical and Physical Sciences, v. 326, pp. 229-255.

Rogers, J.W.R., and Greenberg, J.K., 1990, Late-orogenic, postorogenic, and anorogenic granites: distinction by major element and trace-element chemistry and possible origins: Journal of Geology, v. 98, pp. 291-309.

Rolland, Y., Picard, C., Lapierre, H., Bosch, D., and Keller, F., 2002. The Cretaceous Ladakh arc of NW Himalaya-slab melting and melt mantle interaction during fast northward drift of the Indian Plate: Chemical Geology, v. 182, pp. 139-178.

Roy, P., Jain, and A. K., Singh, S, 2010, Microstructures of Mylonites along the Karakoram Shear Zone, Tangste Valley, Pangong Mountains, Karakoram: Journal Geological Society Of India, v. 75, pp. 679-694

Saini, N.K., Mukherjee, P.K., Rathi, M.S., Khanna, P.P., and Purohit, K.K., 1998, A new geochemical reference sample of granite (DH-G) from Dalhousie Himachal Himalaya: Journal of the Geological Society of India, v. 52, pp. 603-606.

Sawyer, E.W., 2010, Migmatites formed by water-fluxed partial melting of a leucogranodiorite protolith: Microstructures in the residual rocks and source of the fluid: Lithos, v. 116 pp. 273-286

Schärer, U., Copeland, P., Harrison, T.M., and Searle, M.P., 1990, Age, cooling history and origin of post-collisional leucogranites in the Karakoram batholith, a multi-system isotope study: Journal of Geology, v. 98, pp. 233-251.

Schärer, U., Hamet, J., and Allègre, C.J., 1984, The Trans Himalaya (Gangdese) plutonism in the Ladakh region: a $\mathrm{U}-\mathrm{Pb}$ and $\mathrm{Rb}-\mathrm{Sr}$ study: Earth and Planetary Science Letters, v. 67, no.3, pp. 327-339.

Searle, M.P. 1991, Geology and Tectonics of the Karakoram Mountains. J. Wiley \& Sons Ltd. Chichester.
Searle, A.P., Cooper, D.J.W., and Rex, A.J., 1988, Collision tectonics of the Ladakh-Zanskar Himalaya: Philosophical Transaction Royal Society of London, v. A326, pp. 117-150.

Searle, M.P., and Phillips, R.J., 2007, Relationships between right-lateral shear along the Karakoram fault and metamorphism, magmatism, exhumation and uplift: evidence from the K2-Gasherbrum-Pangong ranges, north Pakistan and Ladakh: Journal of Geological Society of London, v. 164, pp. 439-450

Searle, M.P., Weinberg, R. F., and Dunlap, W. J., 1998, Transpressional tectonics along the Karakoram fault zone, northern Ladakh: constraints on Tibetan extrusion, in Holdsworth, R. E., Strachan, R. A. and Dewey, J. F., eds, Continental Transpressional and Transtensional Tectonics: Geological Society of London Special Publication, v. 135, pp. 307-326.

Sen, K., Mukherjee, B.K., and Sachan, H.K., 2009, Field and microstructural analysis of the Pangong granodiorite, Ladakh (NW India): implications for tectonics along the Karakoram Fault Zone: Current Science, v. 96, pp. 1124-1130.

Sheraton, J.W., Ellis, D.J., and Kuehner, S.M., 1985, Rare earth element geochemistry of Archean orthogneisses and evolution of the east Antarctica shield: Bureau of Mineral Resources Journal Australian Geology and Geophysics, v. 9, pp. 207-218.

Slagstad, T., Jamieson, R.A., and Culshaw, N.G., 2005, Formation, crystallisation and migration of melt in the mid-orogenic crust: Muskoka domain migmatites, Grenville Province, Ontario: Journal of Petrology, v. 46, pp. 893-919.

Singh, S., Barley, M.E., and Jain, A.K., 2004, Tale of two migmatites and leucogranite generation within the Himalayan Collisional Zone: Evidences from SHRIMP U-Pb zircon ages from Higher Himalayan Metamorphic Belt and Trans-Himalayan Karakoram Metamorphic Belt, India (abs): 19th Himalaya-Karakoram-Tibet Workshop, 2004, Niseko, Japan. Himalayan Journal of Sciences v. 2 Special Issue 4.

Sinha, A.K., Rai, H., Upadhyay, R., and Chandra, R., 1999, Contribution to the geology of the eastern Karakoram, India: Geological Society of America, Special Paper, v. 328, pp. 33-46.

Srimal, N., Basu, A.R., and Kyser, K.Y., 1987, Tectonics inferences from oxygen isotopes in volcano-plutonic complexes of the India-Asia collision zones, NW India: Tectonics, v. 6, pp. 261-273.

Tarney, J., Saunders, A.D., Mattey, D.P., Wood, D.A., and Marsh, N.G., 1981, Geochemical aspects of back-arc spreading in the Scotia Sea and west Pacific: Philosophical Transaction of Royal Society of London, v. A300, pp. 263-285

ten Grotenhuis, S.M., Trouw, R.A.J. and Passchier, C.W. 2003, Evolution of mica fish in mylonitic rocks: Tectonophysics, v. 372, pp. 1-21.

Treloar, P.J., Petterson, M.G., Jan, M.Q., and Sullivan, M.A., 1996, A reevaluation of the stratigraphy and evolution of the Kohistan arc sequence, Pakistan Himalaya: implications for magmatic and tectonic arc building processes: Journal of the Geological Society of London, v. 153 (5), pp. 681-693

Upadhyay, R., Frisch, W., and Siebel, W., 2008, Tectonic implications of U$\mathrm{Pb}$ zircon ages of the Ladakh batholith, Indus suture zone, northwest Himalaya, India: Terra Nova, v. 20, pp. 309-317.

Virdi, N.S., 1981, Occurrence of paired-metamorphic belts in the Himalaya: Journal of Geological Society of India, v. 22, pp. 547-554.

Weinberg, R.F., and Dunlap, W.J., 2000, Growth and Deformation of the Ladakh Batholith, Northwest Himalayas: implications for timing of continental collision and origin of calc-alkaline batholiths: Journal of Geology, v.108, pp. 303-320

Weinberg, R.F., and Mark, G., 2008, Magma migration, folding, and disaggregation of migmatites in the Karakoram shear zone, Ladakh, NW India: Geological Society of America Bulletin, v. 120, pp. 994-1009.

Weinberg, R.F., Mark, G., and Reichardt, H., 2009, Magma ponding in the Karakoram shear zone, Ladakh, NW India: Geological Society of America Bulletin, v. 121, pp. 278.285.

Weinberg, R F., and Searle, M P., 1998, Pangong Injection Complex, Indian Karakoram: A case of pervasive granite flow through hot viscous crust: Journal of Geological Society of London, v.155, pp. 883-891. 
Wood, D.A., Joron, J.L., and Treuil, M., 1979, A re-appraisal of the use of trace element to classify and discriminate between magma series erupted in different tectonic setting: Earth Planetary Science Letters, v. 45, pp. 326-336 .

Wu, F.Y., Clift, P.D., and Yang, J.H., 2007, Zircon Hf isotopic constraints on

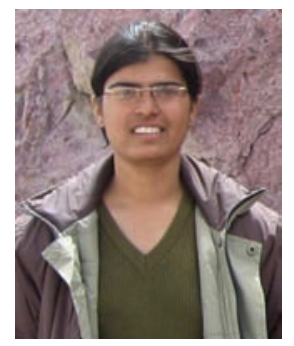

Megha M. Daga is pursuing her doctoral studies at Wadia Institute of Himalayan Geology, Dehradun. She did her M.Sc Geology in 2008 from Solapur University, Maharashtra, India. Her areas of interest are petrology and geochemistry of the magmatic and metamorphic rocks of Ladakh TransHimalaya.

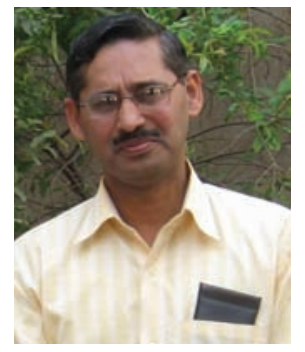

D. Rameshwar Rao had his Honor's Graduation degree in Geology, and M.Tech in Applied Geology. His doctoral work was on the gneiss-charnockite transition rocks of Dharmapuri area, Tamil Nadu, India. Presently, he is working as a senior scientist at Wadia Institute of Himalayan Geology, Dehradun, India. His areas of interest are petrology, geochemistry and geochronology of igneous and metamorphic rocks. the sources of the Indus Molasse, Ladakh Himalaya, India: Tectonics, v. 26, TC2014, pp. 14.

Yin, A., and Harrison, T.M., 2000, Geologic evolution of the HimalayanTibetan orogen: Annual Reviews Earth and Planetary Sciences, v. 28, pp. 211-280.

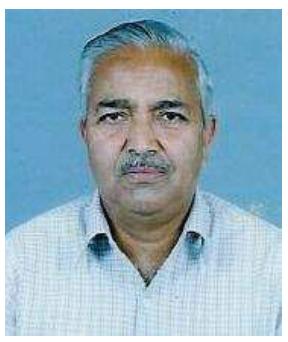

Hakim Rai is presently working as Emeritus Scientist at Wadia Institute of Himalayan Geology, Dehradun, India. He had his graduation, post-graduation and doctoral degrees from the Geology Department, Panjab University, India. He has extensively worked in various parts of the Ladakh TransHimalaya and Karakoram Range. His areas of interest are mapping, petrology of ophiolites and igneous rocks. 